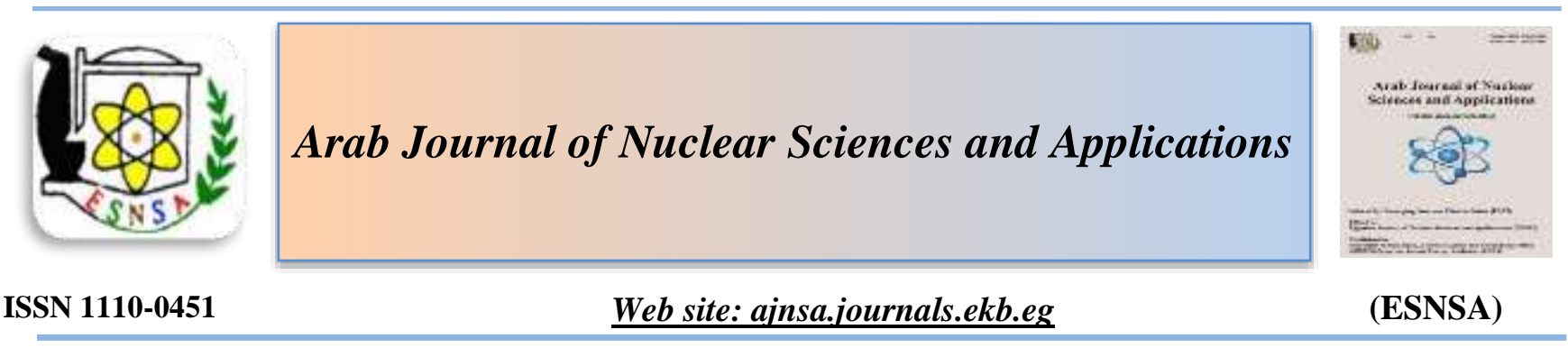

\title{
Investigation of Some Elements Concentrations and Distribution in Body parts and Organs of Japanese Quail Cotornix japonica
}

\author{
*Hassanin W.F. ${ }^{1}$, Abdelaal S.A. ${ }^{2}$, Abdelhady A. M. ${ }^{2}$, Abutaleb A.M. ${ }^{1}$ \\ (1) Biological Applications Department, Radio Isotope Applications Division, Nuclear research Center, \\ Egyptian Atomic Energy Authority \\ (2) Central Lab of Elemental Analysis, Nuclear Research Center, Egyptian Atomic Energy Authority
}

Received 16 Nov 2019 Accepted 15 Jun 2020

\begin{abstract}
The presence, concentration and distribution of 42 trace and ultra-trace elements in addition to rare earth elements (REE), yttrium(Y) and Thorium (Th) were analyzed using Inductively Coupled Plasma Optical Emission Spectrometer (ICP-OES) in different body parts and organs of Japanese quail Cotornix japonica as a laboratory animal model. Twenty one of the investigated elements were below detection limit while the rest of the investigated elements varied in amount and distribution relative to organs and parts in which they were investigated. The existences, concentrations and distributions of thallium (TI) and europium (Er) are recordedin a higher living species in the present work. The present work also documented the presence, concentration and distribution of Thulium (Tm) a living organism. The recorded concentrations and distributions of various elements may be related to their roles in structures and functions of organs in which they were distributed. From the present findings, a better classification of elements according to distribution and concentration levels in individual organs is recommended. It is also recommended to investigate the existence of $\mathrm{Tm}, \mathrm{Tl}$, and $\mathrm{Er}$ in other living species and a further investigation of the role of $\mathrm{Tm}, \mathrm{Tl}$, and $\mathrm{Er}$ in structure and metabolism of Cotornix japonica parts and organs in which they were detected.
\end{abstract}

Keywords: ICP-OES /element distribution/ element concentration/ trace elements/ ultra-trace elements

\section{Introduction}

The roles of many elements in biochemical processes in various biological systems have been studied for decades. It has been shown that the levels of elements are crucial for normal growth and functions of living organisms[1,2,3].Element requirements varies from living organism to another, [4]but in general they can be categorized according to their requirements and distributions into three major groups : Major elements (Oxygen $(\mathrm{O})$, Hydrogen $(\mathrm{H})$, Carbon(C), Nitrogen (N), Phosphorus (Na),Potassium(K), $(\mathrm{Mg})$,Calcium $(\mathrm{Ca})$, and chlorine $(\mathrm{Cl})$ whose concentrations are higher than $0.01 \%$, trace elements (Iron (Fe),Zinc(Zn), Fluorine (F), Strontium(Sr), Molybdenum (Mo),Cupper( $\mathrm{Cu}$, Bromine (Br), Silicon ( $\mathrm{Si}$ ), Cesium(Cs), Iodine (I), Manganese

$(\mathrm{Mn})$,Aluminum

(Al),Lead(Pb),Cadmium(Cd),Boron(B), and Rubidium $(\mathrm{Rb})$ ) having concentrations from $10^{-6} \%$ to $10^{-2} \%$, and ultra-trace elements with concentrations less than $10^{-6} \%$ including Selenium (Se), Cobalt (Co), Vanadium (V), Chromium(Cr),Arsenic(As),Nickel(Ni),

Lithium

(Li),Barium(Ba),Titanium(Ti),Silver(Ag), Tin(Sn), Beryllium(Be),Gallium(Ga), Germanium (Ge),

Corresponding author: walaahassanin@gmail.com

DOI: 10.21608/ajnsa.2020.19474.1298

(C) Scientific Information, Documentation and Publishing Office (SIDPO)-EAEA 
Mercury (Hg),Scandium(Sc), Zirconium (Zr), Bismuth (Bi), Antimony (Sb),Uranium(U),Thorium(Th), and rhodium (Rh) [5]. The major elements $\mathrm{O}, \mathrm{H}, \mathrm{C}, \mathrm{N}, \mathrm{P}$, and $\mathrm{S}$ are referred to as organogens because they contribute in the formation of organs and tissues [5]. Many trace elements such as $\mathrm{Co}, \mathrm{Cu}, \mathrm{Fe}, \mathrm{Mn}, \mathrm{Mo}$, Se and $\mathrm{Zn}$ act as prosthetic groups for many enzymes. Some of the trace elements and ultra-trace elements are known to be essential but their functions are not yet revealed such as Silicon, vanadium, nickel, tin, others are not classified as essential but have effects on human and experimental animals which are fluorine and lithium [6]. Other trace and ultra-trace elements do not have known functions with toxic effect in excess [6]. Studies on the biochemical properties of lanthanides focused on their medicinal applications, toxicity, and their use as informative probes in biological and biochemical research[7].In complex multi-system living organisms, different organs contribute in the normal physiological process of the living organism through specialized activities of their tissue structures that require different levels of trace elements. The knowledge about the essentiality of chemical elements in different forms of life seems to be incomplete. The essential elements are not yet known all with certainty and the knowledge about the functions of apparently non-essential elements is even less than the essential elements[8].In addition to its economic importance as an alternative to commonly used chicken [9], Japanese quail (Cotornix japonica, $\boldsymbol{C}$. japonica for short) are used as Laboratory model animal in many laboratories all over the world ${ }^{[10]}$. Because of its short lifespan combined with its physiological similarity to humans, $C$. japonica has been considered as an ideal model for many studies in physiology, immunology, endocrinology, and reproductive biology[10]. The contents of the elements were determined in male and female Japanese quail fed to captive raptors using atomic absorption of ashed whole quail samples (whole body samples of birds were burned in muffle furnace oven to evaporate all organic matter and leaving only organic elements for analysis). The average levels in $\mathrm{mg} / \mathrm{g}$ of $\mathrm{Cu}, \mathrm{Fe}, \mathrm{Mg}, \mathrm{Mn}, \mathrm{Zn}$, and $\mathrm{Ca}$ recorded for male samples were 2.66, 85.7, $578.6,6.61,55.01$, and 32685 , respectively and for female samples were 3.02, 112.4, 752.7, 8.45, 54.3 and 43615 respectively. The differences between male and female samples in the studied elements levels were not significant according to the author
[11]. In another study [12], the authors, in order to estimate the nutritional requirements of $\boldsymbol{C}$. japonica at different ages, studied the concentrations of $\mathrm{Ca}$, $\mathrm{Mg}, \mathrm{P}, \mathrm{K}, \mathrm{Na}, \mathrm{Cu}, \mathrm{Fe}, \mathrm{Mn}, \mathrm{Ni}, \mathrm{Se}, \mathrm{Zn}$ in ashes of whole birds (digestive contents removed) using atomic absorption. Sales et al. recorded the mean concentrations of $\mathrm{Ca}, \mathrm{Mg}, \mathrm{P}, \mathrm{K}$, and $\mathrm{Na} 223.3 \mathrm{mg} / \mathrm{g}$, $10.85 \mathrm{mg} / \mathrm{g}, 174.9 \mathrm{mg} / \mathrm{g}, 104 \mathrm{mg} / \mathrm{g}$ and $44.14 \mathrm{mg} / \mathrm{g}$, respectively. The concentrations of $\mathrm{Cu}, \mathrm{Fe}, \mathrm{Mn}, \mathrm{Ni}$ and $\mathrm{Zn}$ were 66.7, 1074, 49.03, 39.88, $1115 \mathrm{mg} / \mathrm{kg}$ respectively, while, Se recorded concentration was $8012 \mu \mathrm{g} / \mathrm{g}$ [12]. The concentrations of some heavy elements were determined in edible parts of migratory Japanese quail using ICP-MS. The samples of liver, kidney and muscles (chest \& leg) were analyzed for the presence of $\mathrm{Al}, \mathrm{Cu}, \mathrm{Fe}, \mathrm{Mn}$, $\mathrm{Zn}, \mathrm{Cr}, \mathrm{Mo}, \mathrm{Ni}, \mathrm{Pb}$ and $\mathrm{Sr}$. Their recorded ranges were $\mathrm{Al}$ (48.61 - 395.9), $\mathrm{Cu}(2.84$ - 6.64), $\mathrm{Fe}$ (162.3 - 882.7), Mn (2.18 - 12.2) and Zn (8.11- 25.5), Cr (2.45-14.51), $\mathrm{Sr}$ (1.97-8.65), Pb (1.06-6.17), Ni (0.84-5.42) and Mo (0.23-1.48) $\mathrm{mg} / \mathrm{kg}$ wet weight [13].

The inductively coupled plasma optical emission spectrometer (ICP-OES) is widely used in elemental analysis, because of its ability to obtain low detection limits, highly sensitive technique, its precision and can perform multi element analysis when compared with other analytical techniques [13]. In this technique, emission signal response depends directly on the number of analyte atoms and ions present in plasma which depends mainly on the sample. In ICP-OES, the radiation generated is optically aligned then dispersed and finally measured using an appropriate detector [14, 15].In this study, the inductively coupled plasma optical emission spectrometer (ICP-OES) is used for multielement analyses to determine the reference values of trace, ultra-trace elements and REE, in addition to $\mathrm{Y}$ and $\mathrm{Th}$, in main organs of $C$. japonica which are fed basal feed meal.

\section{Experimental and design}

A total number of $50 \boldsymbol{C}$. japonica chicks, obtained from the farm of Biological Applications Department, Nuclear Research Center, Atomic Energy Authority, Egypt were used in the present study. The quail chicks were randomly selected. They were battery reared and provided with water and feed ad Libitum under the same conditions of temperature and light regimen during the experimental period which lasted for (6 weeks) of age. Quails basal diets contained $3000 \mathrm{Kcal} \mathrm{ME} / \mathrm{Kg}$ 
and 24.25 crude protein in starting periods. Quails basal diets contained 3000 and $2862 \mathrm{Kcal} \mathrm{ME} / \mathrm{Kg}$ and 24.25 and $21.2 \%$ crude protein in Starting and Laying periods, respectively, as shown in Table (1). All chemicals were purchased from Honeywell Fluka.

At the age of six weeks, the birds were slaughtered for blood, part and organ samples collection. Blood samples were maintained in tubes to coagulate, then the serum sample were separated by centrifugation at $3000 \times \mathrm{g}$ for 5 minutes. Blood coagulate samples and selected parts and organs were weighted, freeze-dried, and then dry weights were recorded. $0.5 \mathrm{ml}$ of serum samples and Weights between 0.5 to $1 \mathrm{~g}$ of blood coagulate and dry organ samples were digested using a mixture of concentrated nitric acid and hydrogen peroxide then the sample digests were diluted with de-ionized water to a final volume of $10 \mathrm{ml}$. multi elements analysis of diluted samples were performed using Prodigy High Dispersion Inductively Coupled Plasma Optical Emission Spectrometer (Teledyne Leeman ICP-OES USA). The specific spectral lines of the measured elements are shown in Table (2). O, H, C, and $\mathrm{N}$ were not analyzed in this study because they are components of air which is found in the prism environment. Halogens were not analyzed also because, when exited by plasma, their atoms escape leading high uncertainty in measurements.

\section{Results and Discussion}

The presence and concentration of 42 elements were investigated in a total of 13 samples from each bird. The samples included brain, beak, crop, eyes, gizzard, heart, intestines, skeletal muscles, kidneys, lungs, liver, ovaries in addition to coagulated blood and serum. The elements investigated included trace elements which are $\mathrm{Al}, \mathrm{Cs}, \mathrm{Cu}, \mathrm{Fe}, \mathrm{Pb}, \mathrm{Mn}$, $\mathrm{Mo}, \mathrm{Si}$, and $\mathrm{Sr}$. in addition to Ultra trace elements which are Ag, As, B, Ba, Cr, Co, Li, Ni, Sb, Se, Ti, $\mathrm{Tl}, \mathrm{Th}, \mathrm{V}, \mathrm{W}$, and $\mathrm{Zr}$. The presence and concentration of the rare earth elements (REE) group in addition to Ytterbium and thorium were also investigated. Twenty one of the investigated elements which are $\mathrm{Li}, \mathrm{Be}, \mathrm{As}, \mathrm{V}, \mathrm{Y}, \mathrm{Ag}, \mathrm{Cd}, \mathrm{Cs}$, and all REE except Er and Tm were below the detection limit while the rest of the investigated elements varied in amount and distribution relative to organs and parts in which they were investigated, as summarized in Tables (3-18) and Figures(1-4). Not all detected elements were found in all organs nor they had the same amounts. Types of elements detected also varied from organ to another.

In the $\boldsymbol{C}$. japonica beak, 15trace, ultra-trace elements and REE were detected and determined (Table 3). The highest element in concentration was found to be $\mathrm{Fe}$ in the range of $55.29-499.43 \mu \mathrm{g} / \mathrm{g}$ of dry Weight and had average concentration of $208.91 \pm 18.24 \mu \mathrm{g} / \mathrm{g}$ dry Wt. Fe represented $35.4 \%$ of total detected elements. Tl and Tm were detected in the $\boldsymbol{C}$.japonica beak. $\mathrm{Tl}$ abundance was about $0.3 \%$ in bone tissue while $\mathrm{Tm}$ abundance was $0.2 \%$. To the best of our knowledge, there are no records for the presence of $\mathrm{Tm}$ in living organisms.

The $\boldsymbol{C}$. japonica crop contained 12 elements as shown in Table (5) and as in the brain, Fe was found to have the highest abundance among detected elements. The estimated range of $\mathrm{Fe}$ in the crop is $85.57-173.49 \mu \mathrm{g} / \mathrm{g}$ of dry Weight. and its average concentration is $119.18 \pm 3.37 \mu \mathrm{g} / \mathrm{g}$ dry Wt. which represents $44.13 \%$ of total detected elements. From the recorded trace and ultra-trace elements and lanthanides [1-7], 11 elements were detected [17]. Twelve trace, ultra-trace elements and REE were detected in the $\boldsymbol{C}$. japonica eyes, Table (6). Fe recorded the highest concentration in the $\boldsymbol{C}$. japonica eyes which was $147.21 \pm 46.97 \mu \mathrm{g} / \mathrm{g}$ dry Wt. Fe estimated range was 34.04-448.7 $\mu \mathrm{g} / \mathrm{g}$ of dry Weight and represented $49.25 \%$ of total detected elements. Ba, Th, W, and the REE Er detected concentrations in the $\boldsymbol{C}$. japonica eyes are $2.44 \times 10^{-}$ $3 \% \mathrm{w} / \mathrm{w}, 2.53 \times 10^{-4} \% \mathrm{w} / \mathrm{w}, 1.45 \times 10^{-4} \% \mathrm{w} / \mathrm{w}$. and 5 $\mathrm{x} 10^{-5} \% \mathrm{w} / \mathrm{w}$ respectively. Although they are classified as ultra-trace elements which have concentrations less than $10^{-6} \%$ [5] The determined concentrations of these four elements in the $\boldsymbol{C}$. japonica eyes were found to be in the range of trace elements which is between $10^{-6} \%$ to $10^{-2} \%$ [5].

In the $\boldsymbol{C}$. japonica gizzard, 15trace, ultra-trace elements and REE were detected and determined, Table (7). The highest element in concentration was found to be Fe that ranged 94.17-129.54 and had an average concentration of $112.47 \pm 1.45 \mu \mathrm{g} / \mathrm{g}$ dry Wt. which represented $38.9 \%$ of total detected elements. The ultra-trace elements $\mathrm{Ba}, \mathrm{Cr}, \mathrm{Ni}, \mathrm{Se}$, $\mathrm{Th}, \mathrm{Ti}$, and $\mathrm{W}$ and the REE Er were found to have concentrations of $1.02 \times 10^{-4} \% \mathrm{w} / \mathrm{w}, 2.3 \times 10^{-5} \% \mathrm{w} / \mathrm{w}$, $2.3 \times 10^{-5} \% \mathrm{w} / \mathrm{w}, 1.35 \times 10^{-4} \% \mathrm{w} / \mathrm{w}, 4.5 \times 10^{-5} \% \mathrm{w} / \mathrm{w}$, $1.4 \times 10^{-5} \% \mathrm{w} / \mathrm{w}, 1.5 \times 10^{-4} \% \mathrm{w} / \mathrm{w}$, and $5.3 \times 10^{-5} \%$ $\mathrm{w} / \mathrm{w}$ respectively. The determined concentrations of these elements in the $\boldsymbol{C}$. japonica gizzard were found to be in the range of trace elements which is between $10^{-6} \%$ to $10^{-2} \%$ [5]. 
INVESTIGATION OF SOME ELEMENTS CONCENTRATIONS...

Table (1): components of basal Diet fed to quail chick during experiment

\begin{tabular}{|c|c|c|}
\hline Contents & Starter diet & \\
\hline Ground yellow corn & 53.00 & \\
\hline Soybean meal (44\%) & 34.00 & \\
\hline Corn gluten meal (60\%) & 7.000 & \\
\hline Calcium carbonate & 1.400 & \\
\hline Dicalcium phosphate & 1.600 & \\
\hline Sodium chloride & 0.400 & \\
\hline Vegetable oil & 1.500 & \\
\hline L-Lysine-Hcl & 0.200 & \\
\hline DL-Methionine & 0.280 & \\
\hline Choline chloride & 0.240 & \\
\hline Premix* & & 0.380 \\
\hline Calculated analysis** & 100.00 & \\
\hline Crude protein & 24.0 & \\
\hline ME & 3000 & \\
\hline $\mathrm{Ca}$ & 1.01 & \\
\hline Av.P & 0.45 & \\
\hline Lys. & 1.37 & \\
\hline Met. & 0.64 & \\
\hline Met+Cys & & 1.11 \\
\hline
\end{tabular}

*Each kilogram of diet contains, A, 12000 I.U; $D_{3}, 2000$ I.U; E, 10mg; K, 2mg; $B_{1}, 1 \mathrm{mg} ; B_{6}, 1.5 m g ; B_{12}, 10 \mu g ; B_{2}$, 4mg; Pantothonic

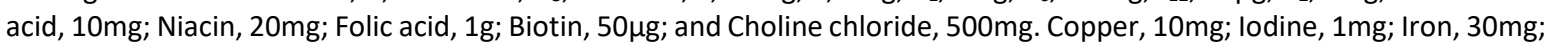
Manganese, 55mg; Zing, 55mg and Selenium, $1 \mathrm{mg}$.

** Values were calculated according to the nutrient composition to the NRC [16]

Table (2): The specific spectral lines of the measured elements

\begin{tabular}{cccc}
\hline Element & Line $(\mathrm{nm})$ & Line type & S/B ratio \\
\hline $\mathrm{Al}$ & 396.152 & $\mathrm{~A}$ & 1050 \\
$\mathrm{Ba}$ & 455.403 & $\mathrm{I}$ & 23000 \\
$\mathrm{Co}$ & 228.615 & $\mathrm{I}$ & 4300 \\
$\mathrm{Cr}$ & 267.716 & $\mathrm{I}$ & 4200 \\
$\mathrm{Cu}$ & 224.700 & $\mathrm{I}$ & 3900 \\
$\mathrm{Er}$ & 337.271 & $\mathrm{I}$ & 2900 \\
$\mathrm{Fe}$ & 240.489 & $\mathrm{I}$ & 2700 \\
$\mathrm{Mn}$ & 257.610 & $\mathrm{I}$ & 22000 \\
$\mathrm{Mo}$ & 277.540 & $\mathrm{I}$ & 1200 \\
$\mathrm{Ni}$ & 232.003 & $\mathrm{~A}$ & 2000 \\
$\mathrm{~Pb}$ & 217.000 & $\mathrm{~A}$ & 330 \\
$\mathrm{Sb}$ & 206.833 & $\mathrm{~A}$ & 910 \\
$\mathrm{Se}$ & 196.090 & $\mathrm{~A}$ & 400 \\
$\mathrm{Si}$ & 288.158 & $\mathrm{~A}$ & 1100 \\
$\mathrm{Sr}$ & 407.77 & $\mathrm{I}$ & 72000 \\
$\mathrm{Th}$ & 283.730 & $\mathrm{I}$ & 460 \\
$\mathrm{Ti}$ & 334.941 & $\mathrm{I}$ & 7900 \\
$\mathrm{Tl}$ & 190.864 & $\mathrm{I}$ & 740 \\
$\mathrm{Tm}$ & 313.126 & $\mathrm{I}$ & 5800 \\
$\mathrm{~W}$ & 207.911 & $\mathrm{I}$ & 1000 \\
$\mathrm{Zn}$ & 202.548 & $\mathrm{I}$ & 7500 \\
$\mathrm{Zr}$ & 339.198 & $\mathrm{I}$ & 3900 \\
\hline
\end{tabular}

$\mathrm{A}=\mathrm{A}=$ Atomic line, $\mathrm{I}=$ Ionic line 
Table (3): mean concentrations, ranges and Abundances of detected Elements in $\boldsymbol{C}$. japonica beak

\begin{tabular}{|c|c|c|c|c|}
\hline Element & $\operatorname{Mean}(\mu \mathrm{g} / \mathrm{g}$ dry Wt. $) \pm \mathrm{SD}$ & Range $(\mu \mathrm{g} / \mathrm{g}$ dry Wt. $)$ & Abundance $(\%)$ & Concentration $\% \mathrm{w} / \mathrm{w}$ \\
\hline $\mathrm{Al}(\mu \mathrm{g} / \mathrm{g}$ dry $\mathrm{Wt})$. & $36.6 \pm 4.17$ & $3.07-96.79$ & 6.2 & $3.66 \times 10^{-3}$ \\
\hline $\mathrm{Ba}(\mu \mathrm{g} / \mathrm{g}$ dry $\mathrm{Wt})$. & $48.6 \pm 9.04$ & $6.055-210.195$ & 8.2 & $4.86 \times 10^{-3}$ \\
\hline Co $(\mu \mathrm{g} / \mathrm{g}$ dry Wt.) & < D.L. & ------- & ----- & ------- \\
\hline $\mathrm{Cr}(\mu \mathrm{g} / \mathrm{g}$ dry $\mathrm{Wt})$. & $0.63 \pm 0.04$ & $0.24-1.18$ & 0.1 & $6.3 \times 10^{-5}$ \\
\hline $\mathrm{Cu}(\mu \mathrm{g} / \mathrm{g}$ dry Wt. $)$ & $4.74 \pm 0.27$ & $2.31-9.25$ & 0.8 & $4.74 \times 10^{-4}$ \\
\hline $\operatorname{Er}(\mu \mathrm{g} / \mathrm{g}$ dry Wt. $)$ & $10.48 \pm 1.65$ & $0.67-39.61$ & 1.8 & $1.05 \times 10^{-3}$ \\
\hline $\mathrm{Fe}(\mu \mathrm{g} / \mathrm{g}$ dry $\mathrm{Wt})$. & $208.91 \pm 18.24$ & $55.29-499.43$ & 35.4 & $2.09 \times 10^{-2}$ \\
\hline Mn $(\mu \mathrm{g} / \mathrm{g}$ dry Wt. $)$ & $14.9 \pm 0.59$ & $8.18-20.22$ & 2.5 & $1.49 \times 10^{-5}$ \\
\hline Mo $(\mu \mathrm{g} / \mathrm{g}$ dry Wt. $)$ & < D.L. & ------- & ------- & ------- \\
\hline Ni ( $\mu \mathrm{g} / \mathrm{g}$ dry Wt.) & $<$ D.L. & ------- & ------- & ------- \\
\hline $\mathrm{Pb}(\mu \mathrm{g} / \mathrm{g}$ dry Wt. $)$ & $<$ D.L. & ------- & ------- & ------- \\
\hline $\mathrm{Sb}(\mu \mathrm{g} / \mathrm{g}$ dry Wt. $)$ & <D.L. & ------- & ------ & ------- \\
\hline $\mathrm{Se}(\mu \mathrm{g} / \mathrm{g}$ dry Wt. $)$ & <D.L. & ------- & ------- & ------- \\
\hline $\mathrm{Si}(\mu \mathrm{g} / \mathrm{g}$ dry Wt. $)$ & $90.43 \pm 5.20$ & $33.99-169.51$ & 15.3 & $9.4 \times 10^{-3}$ \\
\hline $\mathrm{Sr}(\mu \mathrm{g} / \mathrm{g}$ dry Wt. $)$ & $23.46 \pm 0.61$ & $17.59-25.5$ & 4.0 & $2.35 \times 10^{-3}$ \\
\hline Th ( $\mu \mathrm{g} / \mathrm{g}$ dry Wt. $)$ & $3.44 \pm 0.16$ & $2.1-5.55$ & 0.6 & $3.44 \times 10^{-4}$ \\
\hline $\mathrm{Ti}(\mu \mathrm{g} / \mathrm{g}$ dry Wt.) & $5.81 \pm 1$ & $0-23.59$ & 1.0 & $5.81 \times 10^{-4}$ \\
\hline $\mathrm{Tl}(\mu \mathrm{g} / \mathrm{g}$ dry Wt.) & $1.71 \pm 0.17$ & $0-4.67$ & 0.3 & $1.71 \times 10^{-4}$ \\
\hline Tm $(\mu \mathrm{g} / \mathrm{g}$ dry Wt. $)$ & $4.16 \pm 0.47$ & $0-1.33$ & 0.2 & $9.5 \times 10^{-4}$ \\
\hline $\mathrm{W}(\mu \mathrm{g} / \mathrm{g}$ dry $\mathrm{Wt})$. & $2.87 \pm 0.2$ & $1.69-6.42$ & 0.5 & $2.87 \times 10^{-4}$ \\
\hline $\mathrm{Zn}(\mu \mathrm{g} / \mathrm{g}$ dry Wt. $)$ & $136.42 \pm 8.71$ & $78.42-289.53$ & 23.1 & $1.36 \times 10^{-2}$ \\
\hline $\mathrm{Zr}(\mu \mathrm{g} / \mathrm{g}$ dry $\mathrm{Wt})$. & <D.L. & -------- & ------- & -------- \\
\hline
\end{tabular}

Table (4): mean concentrations, ranges and Abundances of detected Elements in $C$. japonica brain

\begin{tabular}{|c|c|c|c|c|}
\hline Element & Mean $(\mu \mathrm{g} / \mathrm{g}$ dry Wt. $) \pm \mathrm{SD}$ & Range $(\mu \mathrm{g} / \mathrm{g}$ dry Wt. $)$ & Abundance (\%) & Concentration $\% \mathrm{w} / \mathrm{w}$ \\
\hline $\mathrm{Al}(\mu \mathrm{g} / \mathrm{g}$ dry Wt.) & $16.32 \pm 2.29$ & $0-55.09$ & 5.7 & $1.63 \times 10^{-3}$ \\
\hline $\mathrm{Ba}(\mu \mathrm{g} / \mathrm{g}$ dry Wt. $)$ & < D.L. & ------ & ------ & ------ \\
\hline Co $(\mu \mathrm{g} / \mathrm{g}$ dry $\mathrm{Wt})$. & < D.L. & ------ & ------ & ------ \\
\hline $\mathrm{Cr}(\mu \mathrm{g} / \mathrm{g}$ dry $\mathrm{Wt})$. & $0.93 \pm 0.12$ & $0.18-3.15$ & 0.3 & $9.3 \times 10^{-5}$ \\
\hline $\mathrm{Cu}(\mu \mathrm{g} / \mathrm{g}$ dry $\mathrm{Wt})$. & $12 \pm 0.36$ & $7.91-16.88$ & 4.2 & $1.2 \times 10^{-3}$ \\
\hline $\operatorname{Er}(\mu \mathrm{g} / \mathrm{g}$ dry Wt. $)$ & $1.46 \pm 0.17$ & $0-4.46$ & 0.5 & $1.46 \times 10^{-4}$ \\
\hline $\mathrm{Fe}(\mu \mathrm{g} / \mathrm{g}$ dry Wt. $)$ & $164.12 \pm 13.84$ & $55.95-401.36$ & 57.2 & $1.64 \times 10^{-2}$ \\
\hline Mn ( $\mu \mathrm{g} / \mathrm{g}$ dry Wt.) & $1.77 \pm 0.08$ & $1.06-3.14$ & 0.6 & $1.77 \times 10^{-4}$ \\
\hline Mo ( $\mu \mathrm{g} / \mathrm{g}$ dry Wt.) & < D.L. & ------ & ------ & ------ \\
\hline Ni ( $\mu \mathrm{g} / \mathrm{g}$ dry Wt.) & $<$ D.L. & ----- & ----- & ------ \\
\hline $\mathrm{Pb}(\mu \mathrm{g} / \mathrm{g}$ dry $\mathrm{Wt})$. & $<$ D.L. & ------ & ----- & ------ \\
\hline $\mathrm{Sb}(\mu \mathrm{g} / \mathrm{g}$ dry $\mathrm{Wt})$. & $<$ D.L. & ----- & ------ & ------ \\
\hline Se $(\mu \mathrm{g} / \mathrm{g}$ dry Wt. $)$ & < D.L. & ------ & ------ & ------ \\
\hline Si ( $\mu \mathrm{g} / \mathrm{g}$ dry Wt.) & $46.2 \pm 7.55$ & $0-178.35$ & 16.1 & $4.62 \times 10^{-3}$ \\
\hline $\mathrm{Sr}(\mu \mathrm{g} / \mathrm{g}$ dry Wt. $)$ & < D.L. & ------ & ---- & ------ \\
\hline Th ( $\mu \mathrm{g} / \mathrm{g}$ dry Wt.) & $<$ D.L. & ------ & ------ & ------ \\
\hline $\mathrm{Ti}(\mu \mathrm{g} / \mathrm{g}$ dry Wt. & $<$ D.L. & ------ & - & ----- \\
\hline $\mathrm{Tl}(\mu \mathrm{g} / \mathrm{g}$ dry Wt. $)$ & $<$ D.L. & ----- & ------ & ------ \\
\hline Tm ( $\mu \mathrm{g} / \mathrm{g}$ dry Wt. $)$ & < D.L. & ------ & ------ & ------ \\
\hline W ( $\mu \mathrm{g} / \mathrm{g}$ dry Wt.) & $0.94 \pm 0.02$ & $0.65-1.3$ & 0.3 & $9.4 \times 10^{-5}$ \\
\hline $\mathrm{Zn}(\mu \mathrm{g} / \mathrm{g}$ dry Wt. $)$ & $42.93 \pm 0.97$ & $32.64-54.02$ & 15 & $4.29 \times 10^{-3}$ \\
\hline $\mathrm{Zr}(\mu \mathrm{g} / \mathrm{g}$ dry Wt. $)$ & < D.L. & ----- & ----- & ------ \\
\hline
\end{tabular}


Table (5): mean concentrations, ranges and Abundances of detected Elements in $C$. japonica Crop

\begin{tabular}{|c|c|c|c|c|}
\hline Element & Mean $(\mu \mathrm{g} / \mathrm{g}$ dry Wt. $) \pm \mathrm{SD}$ & Range ( $\mu \mathrm{g} / \mathrm{g}$ dry Wt.) & Abundance (\%) & Concentration $\% \mathrm{w} / \mathrm{w}$ \\
\hline $\mathrm{Al}(\mu \mathrm{g} / \mathrm{g}$ dry Wt.) & $26.58 \pm 3.2$ & $0-71.36$ & 8.74 & $2.66 \times 10^{-3}$ \\
\hline $\mathrm{Ba}(\mu \mathrm{g} / \mathrm{g}$ dry $\mathrm{Wt})$. & $1.37 \pm 0.12$ & $0-3.42$ & 0.45 & $1.36 \times 10^{-4}$ \\
\hline Co $(\mu \mathrm{g} / \mathrm{g}$ dry $\mathrm{Wt})$. & < D.L. & ------ & ----- & ------- \\
\hline $\mathrm{Cr}(\mu \mathrm{g} / \mathrm{g}$ dry Wt. $)$ & $0.31 \pm 0.01$ & $0.25-0.36$ & 0.10 & $3.1 \times 10^{-5}$ \\
\hline $\mathrm{Cu}(\mu \mathrm{g} / \mathrm{g}$ dry $\mathrm{Wt})$. & $10.98 \pm 0.16$ & $8.26-12.37$ & 3.61 & $1.1 \times 10^{-3}$ \\
\hline $\operatorname{Er}(\mu \mathrm{g} / \mathrm{g}$ dry Wt. $)$ & <D.L. & ------ & ------ & ------- \\
\hline $\mathrm{Fe}(\mu \mathrm{g} / \mathrm{g}$ dry $\mathrm{Wt})$. & $119.18 \pm 3.37$ & $85.57-173.49$ & 39.20 & $1.19 \times 10^{-2}$ \\
\hline Mn $(\mu \mathrm{g} / \mathrm{g}$ dry Wt. $)$ & $7.46 \pm 0.26$ & $4.24-11.27$ & 2.45 & $7.46 \times 10^{-4}$ \\
\hline Mo $(\mu \mathrm{g} / \mathrm{g}$ dry Wt. $)$ & < D.L. & ------ & ----- & ------- \\
\hline $\mathrm{Ni}(\mu \mathrm{g} / \mathrm{g}$ dry Wt. & $<$ D.L. & ----- & ------ & ------- \\
\hline $\mathrm{Pb}(\mu \mathrm{g} / \mathrm{g}$ dry $\mathrm{Wt})$. & < D.L. & ----- & ----- & ------- \\
\hline $\mathrm{Sb}(\mu \mathrm{g} / \mathrm{g}$ dry $\mathrm{Wt})$. & < D.L. & ------ & ------ & ------- \\
\hline Se $(\mu \mathrm{g} / \mathrm{g}$ dry Wt. $)$ & $2.02 \pm 0.06$ & $1.57-2.85$ & 0.66 & $2.02 \times 10^{-4}$ \\
\hline $\mathrm{Si}(\mu \mathrm{g} / \mathrm{g}$ dry Wt.) & $41.62 \pm 1.56$ & $23.41-62.28$ & 13.69 & $4.16 \times 10^{-3}$ \\
\hline $\mathrm{Sr}(\mu \mathrm{g} / \mathrm{g}$ dry $\mathrm{Wt})$. & $3.4 \pm 0.12$ & $1.19-4.21$ & 1.12 & $3.4 \times 10^{-4}$ \\
\hline Th $(\mu \mathrm{g} / \mathrm{g}$ dry $\mathrm{Wt})$. & $0.40 \pm 0.02$ & $0-0.71$ & 0.13 & $4 \times 10^{-5}$ \\
\hline $\mathrm{Ti}(\mu \mathrm{g} / \mathrm{g}$ dry Wt. $)$ & < D.L. & ------ & ----- & ------- \\
\hline $\mathrm{Tl}(\mu \mathrm{g} / \mathrm{g}$ dry $\mathrm{Wt})$. & < D.L. & ----- & ----- & ------- \\
\hline Tm ( $\mu \mathrm{g} / \mathrm{g}$ dry Wt. $)$ & < D.L. & ------ & ------ & ------- \\
\hline $\mathrm{W}(\mu \mathrm{g} / \mathrm{g}$ dry Wt.) & $1.55 \pm 0.03$ & 1.24-1.97 & 0.51 & $1.55 \times 10^{-4}$ \\
\hline $\mathrm{Zn}(\mu \mathrm{g} / \mathrm{g}$ dry $\mathrm{Wt})$. & $89.38 \pm 1.52$ & $72.41-113.45$ & 29.40 & $8.94 \times 10^{-3}$ \\
\hline $\mathrm{Zr}(\mu \mathrm{g} / \mathrm{g}$ dry Wt. $)$ & < D.L. & ----- & ----- & ------ \\
\hline
\end{tabular}

Table (6): mean concentrations, ranges and Abundances of detected Elements in $\boldsymbol{C}$. japonica Eyes

\begin{tabular}{ccccc}
\hline Element & Mean( $\mu \mathrm{g} / \mathrm{g}$ dry Wt. $) \pm$ SD & Range $(\mu \mathrm{g} / \mathrm{g}$ dry Wt. $)$ & Abundance $(\%)$ & Concentration \% w/w \\
\hline $\mathrm{Al}(\mu \mathrm{g} / \mathrm{g}$ dry Wt. $)$ & $4.76 \pm 1$ & $0-11.4$ & 1.59 & $4.76 \times 10^{-4}$ \\
$\mathrm{Ba}(\mu \mathrm{g} / \mathrm{g}$ dry Wt. $)$ & $24.38 \pm 0.43$ & $19.84-30.45$ & 8.16 & $2.44 \times 10^{-3}$ \\
$\mathrm{Co}(\mu \mathrm{g} / \mathrm{g}$ dry Wt. $)$ & $<\mathrm{D} . \mathrm{L}$. & ----- & ----- & $--.1 \times 10^{-5}$ \\
$\mathrm{Cr}(\mu \mathrm{g} / \mathrm{g}$ dry Wt. & $0.31 \pm 0.01$ & $0.19-0.52$ & 0.10 & $2.97 \times 10^{-4}$ \\
$\mathrm{Cu}(\mu \mathrm{g} / \mathrm{g}$ dry Wt. $)$ & $2.97 \pm 0.09$ & $2.16-4.31$ & 0.99 & $5 \times 10^{-5}$ \\
$\mathrm{Er}(\mu \mathrm{g} / \mathrm{g}$ dry Wt. $)$ & $0.5 \pm 0.03$ & $0-0.74$ & 0.16 & $1.47 \times 10^{-2}$ \\
$\mathrm{Fe}(\mu \mathrm{g} / \mathrm{g}$ dry Wt. $)$ & $147.21 \pm 46.97$ & $34.04-448.7$ & 49.25 & \\
\hline
\end{tabular}

Table (6) continued: mean concentrations, ranges and Abundances of detected Elements in $\boldsymbol{C}$. japonica Eyes

\begin{tabular}{|c|c|c|c|c|}
\hline Element & Mean $(\mu \mathrm{g} / \mathrm{g}$ dry Wt. $) \pm \mathrm{SD}$ & Range( $\mu \mathrm{g} / \mathrm{g}$ dry Wt. $)$ & Abundance (\%) & Concentration $\% \mathrm{w} / \mathrm{w}$ \\
\hline Mn ( $\mu \mathrm{g} / \mathrm{g}$ dry Wt. $)$ & $1.83 \pm 0.07$ & $1.15-2.96$ & 0.61 & $1.83 \times 10^{-4}$ \\
\hline Mo ( $\mu \mathrm{g} / \mathrm{g}$ dry Wt.) & < D.L. & ------ & ----- & ------- \\
\hline Ni ( $\mu \mathrm{g} / \mathrm{g}$ dry Wt.) & $<$ D.L. & ------ & ------ & ------- \\
\hline $\mathrm{Pb}(\mu \mathrm{g} / \mathrm{g}$ dry $\mathrm{Wt})$. & $<$ D.L. & ----- & ------ & ------- \\
\hline $\mathrm{Sb}(\mu \mathrm{g} / \mathrm{g}$ dry $\mathrm{Wt})$. & $<$ D.L. & ------ & ------ & ------- \\
\hline Se $(\mu \mathrm{g} / \mathrm{g}$ dry Wt. $)$ & $<$ D.L. & ------ & ------ & ------- \\
\hline Si ( $\mu$ g/g dry Wt.) & $34.45 \pm 4.12$ & $0-98.97$ & 11.52 & $3.45 \times 10^{-3}$ \\
\hline $\mathrm{Sr}(\mu \mathrm{g} / \mathrm{g}$ dry Wt. $)$ & $10.54 \pm 0.21$ & $9.16-14.1$ & 3.52 & $1.05 \times 10^{-3}$ \\
\hline Th ( $\mu \mathrm{g} / \mathrm{g}$ dry Wt. & $2.53 \pm 0.03$ & $2.23-2.81$ & 0.85 & $2.53 \times 10^{-4}$ \\
\hline Ti ( $\mu \mathrm{g} / \mathrm{g}$ dry Wt.) & < D.L. & ---- & ----- & ------ \\
\hline $\mathrm{Tl}(\mu \mathrm{g} / \mathrm{g}$ dry Wt. $)$ & $<$ D.L. & ------ & ------ & ------- \\
\hline Tm ( $\mu \mathrm{g} / \mathrm{g}$ dry Wt. $)$ & < D.L. & ------ & ----- & -------- \\
\hline $\mathrm{W}(\mu \mathrm{g} / \mathrm{g}$ dry Wt. $)$ & $1.45 \pm 0.05$ & $0.87-1.25$ & 0.48 & $1.45 \times 10^{-4}$ \\
\hline $\mathrm{Zn}(\mu \mathrm{g} / \mathrm{g}$ dry $\mathrm{Wt})$. & $68.02 \pm 1.37$ & $52.22-88.21$ & 22.76 & $6.8 \times 10^{-3}$ \\
\hline $\mathrm{Zr}(\mu \mathrm{g} / \mathrm{g}$ dry Wt. $)$ & < D.L. & ----- & ----- & \\
\hline
\end{tabular}


Table (7): mean concentrations, ranges and Abundances of detected Elements in $C$. japonica Gizzard

\begin{tabular}{|c|c|c|c|c|}
\hline Element & Mean $(\mu \mathrm{g} / \mathrm{g}$ dry Wt. $) \pm \mathrm{SD}$ & Range $(\mu \mathrm{g} / \mathrm{g}$ dry Wt. $)$ & Abundance (\%) & Concentration $\% \mathrm{w} / \mathrm{w}$ \\
\hline $\mathrm{Al}(\mu \mathrm{g} / \mathrm{g}$ dry Wt. $)$ & $11.72 \pm 0.29$ & $8.34-15.38$ & 4.0 & $1.17 \times 10^{-3}$ \\
\hline $\mathrm{Ba}(\mu \mathrm{g} / \mathrm{g}$ dry $\mathrm{Wt})$. & $1.02 \pm 0.03$ & $0.66-1.29$ & 0.4 & $1.02 \times 10^{-4}$ \\
\hline Co $(\mu \mathrm{g} / \mathrm{g}$ dry Wt. $)$ & < D.L. & ------ & ------ & ------- \\
\hline $\mathrm{Cr}(\mu \mathrm{g} / \mathrm{g}$ dry Wt. $)$ & $0.23 \pm 0.01$ & $0.19-0.31$ & 0.1 & $2.3 \times 10^{-5}$ \\
\hline $\mathrm{Cu}(\mu \mathrm{g} / \mathrm{g}$ dry $\mathrm{Wt})$. & $13.97 \pm 0.29$ & $9.64-16.72$ & 4.8 & $1.4 \times 10^{-4}$ \\
\hline $\operatorname{Er}(\mu \mathrm{g} / \mathrm{g}$ dry Wt. $)$ & $0.53 \pm 0.02$ & $0.28-0.72$ & 0.2 & $5.3 \times 10^{-5}$ \\
\hline $\mathrm{Fe}(\mu \mathrm{g} / \mathrm{g}$ dry Wt. $)$ & $112.47 \pm 1.45$ & $94.17-129.54$ & 38.9 & $1.13 \times 10^{-2}$ \\
\hline Mn ( $\mu \mathrm{g} / \mathrm{g}$ dry Wt.) & $10.29 \pm 0.35$ & $6.23-14.16$ & 3.6 & $1.03 \times 10^{-3}$ \\
\hline Mo ( $\mu \mathrm{g} / \mathrm{g}$ dry Wt.) & < D.L. & ----- & ----- & ------ \\
\hline Ni ( $\mu \mathrm{g} / \mathrm{g}$ dry Wt.) & $0.23 \pm 0.02$ & $0-0.43$ & 0.1 & $2.3 \times 10^{-5}$ \\
\hline $\mathrm{Pb}(\mu \mathrm{g} / \mathrm{g}$ dry $\mathrm{Wt})$. & < D.L. & ----- & ----- & ------ \\
\hline $\mathrm{Sb}(\mu \mathrm{g} / \mathrm{g}$ dry $\mathrm{Wt})$. & $<$ D.L. & ------ & ------ & ------- \\
\hline Se $(\mu \mathrm{g} / \mathrm{g}$ dry Wt.) & $1.35 \pm 0.03$ & $1-1.82$ & 0.5 & $1.35 \times 10^{-4}$ \\
\hline $\mathrm{Si}(\mu \mathrm{g} / \mathrm{g}$ dry Wt.) & $31.34 \pm 1.03$ & $21.43-47$ & 10.8 & $3.13 \times 10^{-3}$ \\
\hline $\mathrm{Sr}(\mu \mathrm{g} / \mathrm{g}$ dry Wt. $)$ & $5.8 \pm 0.38$ & $3-12.1$ & 2.0 & $5.8 \times 10^{-4}$ \\
\hline Th $(\mu \mathrm{g} / \mathrm{g}$ dry Wt. $)$ & $0.45 \pm 0.01$ & $0.35-0.57$ & 0.2 & $4.5 \times 10^{-5}$ \\
\hline Ti ( $\mu \mathrm{g} / \mathrm{g}$ dry Wt. $)$ & $0.14 \pm 0.013$ & $0-0.29$ & 0.05 & $1.4 \times 10^{-5}$ \\
\hline $\mathrm{Tl}(\mu \mathrm{g} / \mathrm{g}$ dry Wt.) & < D.L. & ----- & ----- & ------ \\
\hline $\operatorname{Tm}(\mu \mathrm{g} / \mathrm{g}$ dry Wt. & < D.L. & ------ & ----- & ------ \\
\hline $\mathrm{W}(\mu \mathrm{g} / \mathrm{g}$ dry Wt.) & $1.5 \pm 0.03$ & $1.24-1.86$ & 0.5 & $1.5 \times 10^{-4}$ \\
\hline $\mathrm{Zn}(\mu \mathrm{g} / \mathrm{g}$ dry Wt. $)$ & $98.3 \pm 1.62$ & $84.33-122.98$ & 34.0 & $9.83 \times 10^{-3}$ \\
\hline $\mathrm{Zr}(\mu \mathrm{g} / \mathrm{g}$ dry Wt. $)$ & <D.L. & ------ & ------ & ------- \\
\hline
\end{tabular}

The heart samples of $\boldsymbol{C}$. japonica were found to contain 11trace, ultra-trace elements and REE, Table (8). The highest element in concentration was found to be Fe that ranged from 254.39 to 305.9 and had average concentration of $275.82 \pm 2.6 \mu \mathrm{g} / \mathrm{g}$ dry Wt. which represented $66.18 \%$ of total detected elements. The ultra-trace elements $\mathrm{Ba}, \mathrm{Cr}, \mathrm{Se}$, and $\mathrm{W}$ were found to have concentrations of $1.3 \times 10^{-4} \%$ $\mathrm{w} / \mathrm{w}, 2.5 \times 10^{-5} \% \mathrm{w} / \mathrm{w}, 1.49 \times 10^{-4} \% \mathrm{w} / \mathrm{w}$, and $1.74 \times 10^{-}$ ${ }^{4} \% \quad \mathrm{w} / \mathrm{w}$ respectively. The determined concentrations of these elements in the $\boldsymbol{C}$. japonica heart were found to be in the range of trace elements which is between $10^{-6} \%$ and $10^{-2} \%$ [5].

In the $\boldsymbol{C}$. japonica Intestines, 16trace, ultra trace elements and REE were detected and determined, Table (9). The highest element in concentration was found to be Fe that ranged 142.01-281.23 and had an average concentration of $210.3 \pm 6.64 \mu \mathrm{g} / \mathrm{g}$ dry Wt. which represented $37.32 \%$ of total detected elements. The ultra-trace elements $\mathrm{Ba}, \mathrm{Co}, \mathrm{Cr}, \mathrm{Ni}$, $\mathrm{Sb}, \mathrm{Se}, \mathrm{Th}$, and $\mathrm{W}$ and the REE Er were found to have concentrations of $6.76 \times 10^{-4} \% \mathrm{w} / \mathrm{w}, 7 \times 10^{-5} \%$ $\mathrm{w} / \mathrm{w}, 7.92 \times 10^{-4} \% \mathrm{w} / \mathrm{w}, 3.39 \times 10^{-4} \% \mathrm{w} / \mathrm{w}, 5.5 \times 10^{-}$ $5 \% \mathrm{w} / \mathrm{w}, 8.8 \times 10^{-5} \% \mathrm{w} / \mathrm{w}, 1.23 \times 10^{-4} \% \mathrm{w} / \mathrm{w}, 2.54 \times 10^{-}$ ${ }^{4} \% \mathrm{w} / \mathrm{w}$, and $1.41 \times 10^{-4} \% \mathrm{w} / \mathrm{w}$ respectively. The determined concentrations of these elements in the C. japonica intestines were found to be in the range of trace elements which is between $10^{-6} \%$ to $10^{-2} \%$ [5].

The Kidneys samples of $\boldsymbol{C}$. japonica were found to contain 12trace, ultra trace elements and REE, Table (10). The highest element in concentration was found to be Fe that ranged 206.32-327.25 and had an average concentration of $267.89 \pm 4.49 \mu \mathrm{g} / \mathrm{g}$ dry Wt. which represented $66.04 \%$ of total detected elements. The ultra-trace elements $\mathrm{Cr}, \mathrm{Se}$, and $\mathrm{W}$ were found to have concentrations of $2.9 \times 10^{-5} \mathrm{w} / \mathrm{w}$, $4.47 \times 10^{-4} \% \mathrm{w} / \mathrm{w}$, and $1.17 \times 10^{-4} \% \mathrm{w} / \mathrm{w}$ respectively. The determined concentrations of these elements in the $\boldsymbol{C}$. japonica kidneys were found to be in the range of trace elements which is between $10^{-6 \%}$ to $10^{-2} \%$ [5].

In the $\boldsymbol{C}$. japonica Lungs, 11 trace, ultra trace elements and REE were detected and determined, Table (11). The highest element in concentration was found to be Fe that ranged from 545.06 to 808.6 and had an average concentration of $676.83 \pm 18.64 \mu \mathrm{g} / \mathrm{g}$ dry Wt. which represented $78.35 \%$ of total detected elements. The ultra trace elements $\mathrm{Se}, \mathrm{Th}$, and $\mathrm{W}$ and the REE Er were found to have concentrations of $9.2 \times 10^{-5} \% \mathrm{w} / \mathrm{w}, 2.2 \times 10^{-5} \%$ $\mathrm{w} / \mathrm{w}, 9.9 \times 10^{-5} \% \mathrm{w} / \mathrm{w}, 8.9 \times 10^{-5} \% \mathrm{w} / \mathrm{w}$, respectively. The determined concentrations of these elements with respect to the organ weight of $\boldsymbol{C}$. japonica 
lungs were found to be in the range of trace elements which is between $10^{-6} \%$ to $10^{-2} \%$ [5].

The Liver samples of $\boldsymbol{C}$. japonica were found to contain 14 trace, ultra-trace elements and REE, Table (12). The highest element in concentration was found to be Fe that ranged from 206.32 to 327.25 and had an average concentration of $267.89 \pm 4.49 \mu \mathrm{g} / \mathrm{g}$ dry Wt. which represented $66.04 \%$ of total detected elements. The ultra-trace elements $\mathrm{Cr}, \mathrm{Sb}, \mathrm{Se}, \mathrm{Th}$ and $\mathrm{W}$ were found to have concentrations of $0.22 \times 10^{-5} \% \mathrm{w} / \mathrm{w}, 4.5 \times 10^{-5} \% \mathrm{w} / \mathrm{w}$, $2.26 \times 10^{-4} \% \mathrm{w} / \mathrm{w}, 1.2 \times 10^{-5} \% \mathrm{w} / \mathrm{w}$ and $1.3 \times 10^{-4} \%$ $\mathrm{w} / \mathrm{w}$ respectively. The determined concentrations of these elements with respect to the organ weight of the $\boldsymbol{C}$. japonica Liver were found to be in the range of trace elements which is between $10^{-6} \%$ to $10^{-2} \%$ [5]. The ovaries samples of $\boldsymbol{C}$. japonica were found to contain 10 trace, ultra-trace elements and REE, Table (13). The highest element in concentration was found to be $\mathrm{Zn}$ that ranged from 42.51 to 55.68 and had an average concentration of $52 \pm 3.47 \mu \mathrm{g} / \mathrm{g}$ dry Wt. which represented $48.28 \%$ of total detected elements. The ultra-trace elements $\mathrm{Cr}, \mathrm{Ni}, \mathrm{Sb}, \mathrm{Se}$ and $\mathrm{W}$ were found to have concentrations of 1.4 $\mathrm{x} 10^{-5} \% \mathrm{w} / \mathrm{w}, \quad 2.32 \times 10^{-4} \% \quad \mathrm{w} / \mathrm{w}, 2 \quad \times 10^{-5} \% \mathrm{w} / \mathrm{w}$ , $1.33 \times 10^{-4} \% \mathrm{w} / \mathrm{w}$ and $9.6 \times 10^{-5}$ respectively. The determined concentrations of these elements with respect to the organ weight of the $\boldsymbol{C}$. japonica Liver were found to be in the range of trace elements which is between $10^{-6} \%$ to $10^{-2} \%$ [5].

In the $\boldsymbol{C}$. japonica Skeletal Muscles, 7 trace, ultratrace elements and REE were detected and determined, Table (14). The highest element in concentration was found to be Fe that ranged from $\underline{3.62}$ to $8.76 \mathrm{and}$ had an average concentration of $5.81 \pm 0.08 \mu \mathrm{g} / \mathrm{g}$ dry Wt. which represented $86.69 \%$ of total detected elements. The ultra trace elements $\mathrm{Se}$ and $\mathrm{Tl}$ were found to have concentrations of $9.2 \times 10^{-5} \% \mathrm{w} / \mathrm{wand} 0.41 \times 10^{-5} \% \mathrm{w} / \mathrm{w}$, respectively. The determined concentrations of these elements with respect to the organ weight of $\boldsymbol{C}$. japonica muscles were found to be in the range of trace elements which is between $10^{-6} \%$ to $10^{-2} \%$ [5].

In the $\boldsymbol{C}$. japonica Coagulated blood, 7 trace, ultra-trace elements and REE were detected and determined, table (15). The highest element in concentration was found to be $\mathrm{Fe}$ that ranged from 223.47to 321.49and had an average concentration of $275.64 \pm 4.8 \mu \mathrm{g} / \mathrm{g}$ dry Wt. which represented $95.5 \%$ of total detected elements. The ultra-trace elements $\mathrm{Se}$ and $\mathrm{Tl}$ were found to have concentrations of $5.4 \times 10^{-5} \mathrm{w} /$ wand $9.1 \times 10^{-5} \%$ $\mathrm{w} / \mathrm{w}$, respectively. The determined concentrations of these elements with respect to the organ weight of $\boldsymbol{C}$.japonica muscles were found to be in the range of trace elements which is between $10^{-6} \%$ to $10^{-2} \%$ [5]. The blood serum samples of C. japonica were found to contain 6 trace, ultratrace elements and REE, Table (16). The highest element in concentration was found to be $\mathrm{Fe}$ that ranged from 2.84 to 16.12 and had an average concentration of $10.1 \pm 0.67 \mu \mathrm{g} / \mathrm{g}$ dry Wt. which represented $48.28 \%$ of total detected elements. From the six detected elements, four ultra-trace elements were detected which are $\mathrm{Th}, \mathrm{Tl}, \mathrm{W}$ and $\mathrm{Zr}$ and were found to have concentrations of $2.4 \times 10^{-5}$ $\% \mathrm{w} / \mathrm{w}, 2.3 \times 10^{-5} \%$ w/w,3 $\times 10^{-5} \% \mathrm{w} / \mathrm{wand} 6.2 \times 10^{-}$ ${ }^{4}$ respectively. The determined concentrations of these elements with respect to the organ weight of the $\boldsymbol{C}$. japonica blood serum were found to be in the range of trace elements which is between $10^{-6} \%$ to $10^{-2} \%$ [5]. The concentrations in terms of $\mu \mathrm{g} / \mathrm{g}$ of all measured elements are summarized in Table (17) which showed that the most abundant detected element in studied organs and parts was $\mathrm{Fe}$ which was found to have the highest distribution in body parts and organs and its mean concentration was found to be uppermost in eleven of the studied organs and parts which are the brain, crop, gizzard, heart, intestines, kidneys, lungs and ovaries, Fig. (1). In the beak and eyes $\mathrm{Ba}$ had the highest concentration. The highest number of detected element types was in intestines which contained twenty-one detectable elements, Table (9), and the lowest number of detected element types was ten which was in blood serum, Table (16). On the other hand, the lungs were found to have the highest total mean concentration of element content which was $863.85 \mu \mathrm{g} / \mathrm{g}$ while the lowest recorded organ in total mean concentration of element content was the serum which had mean total element concentration of $12.96 \mu \mathrm{g} / \mathrm{g}$. There are types of elements which were detected in only one organ, namely, Co in intestines and $\mathrm{Tm}$ in Beak. Other elements were detected in two or three organs and body parts, Mo in kidneys and liver, $\mathrm{Pb}$ in liver and coagulated blood, $\mathrm{Ti}$ in gizzard and beak, $\mathrm{Ni}$ in Gizzard intestines and Gonads, $\mathrm{Zr}$ in skeletal muscles, coagulated blood and blood serum. Sb was detected in four organs only which were intestines, liver, kidneys and gonads. $\mathrm{Tl}$ was also detected in only four organs which were beak, skeletal muscles, 
coagulated blood and blood serum. The presence of some detected elements was not recorded as normal elemental content in biological systems these elements are $\mathrm{Tm}, \mathrm{Tl}$, and $\mathrm{Er}{ }^{[2,4,6]}$. Er was found mainly in the bird gastro intestinal tract (GIT) parts which are Beak, intestines and gizzard with mean concentrations $10.5,1.5,0.53 \mathrm{ppm}$ respectively. It was also detected in the brain and liver with mean concentrations of $1.5 \mathrm{ppm}$ in the brain, which is the same as in intestines, and $0.1 \mathrm{ppm}$ in the liver. $\mathrm{Er}$ distribution in the analyzed parts and organs suggests that it may have a function that is not yet studied in the $\boldsymbol{C}$. japonica body parts and organs in which it was concentrated. $\mathrm{Tl}$ was also detected in Beak with a relatively noticeable mean concentration of $1.7 \mathrm{ppm}$, in addition to its presence of coagulated blood, serum and skeletal muscles with mean concentrations $0.91,0.23$ and $0.41 \mathrm{ppm}$ respectively. Tl detected distribution suggests that it may be absorbed and circulated in the blood stream of $\boldsymbol{C}$. japonica with possible contribution in the metabolic and physiological functions of skeletal muscles which is not yet known. Tm was only found in beak with mean concentration of $0.95 \mathrm{ppm}$, with no detectable amounts at any other part of the GIT, this suggest that Tm may accumulate in the beak but its role as a component in this body part it yet to be discovered.

The present findings and results showed that although elements are generally classified into macro trace and ultra-trace elements according to their concentration relative to total body weight ${ }^{[5]}$, this is not the case if the body organs and parts were analyzed individually. It was found that on organ level, some elements levels concentrations were higher than their recorded range of level ranges as in the case of $\mathrm{Cr}$ which is classified as ultra-trace element with a concentration below $10^{-6} \%$ [5]. However, it was found that its measured levels were in the range of trace elements that ranged from $10^{-6}$ $\%$ to $10^{-2} \%$ [5]. Concentrations of individual elements also varied from one organ to another as in the case of some macro elements that recorded levels at the range of trace elements as in the case of $\mathrm{Mg}$ in blood components. It was also detected the noticeable levels of $\mathrm{Tm}, \mathrm{Tl}$, and $\mathrm{Er}$ which are not recorded to be normally present in higher bioorganisms. These elements were detected in specific organs.

\section{Conclusions}

The present study recorded the distribution of 47 elements in various parts and organs of $\boldsymbol{C}$. japonica as a laboratory model animal. The above recorded concentrations and distributions of various elements may be related to their roles in structures and functions of organs in which they were distributed with various levels. According to the above findings, a deeper classification of elements according to distribution and concentration levels in individual organs is recommended. We recommend investigation of the existence of $\mathrm{Tm}, \mathrm{Tl}$, and $\mathrm{Er}$ in other living species and a further investigation of the role of $\mathrm{Tm}, \mathrm{Tl}$, and $\mathrm{Er}$ in structure and metabolism of Cotornix japonica parts and organs in which they were detected.

Table (8): mean concentrations, ranges and Abundances of detected Elements in $C$. japonica Heart

\begin{tabular}{|c|c|c|c|c|}
\hline Element & $\operatorname{Mean}(\mu \mathrm{g} / \mathrm{g}$ dry Wt. $) \pm \mathrm{SD}$ & Range $(\mu \mathrm{g} / \mathrm{g}$ dry Wt. $)$ & Abundance (\%) & Concentration \% w/w \\
\hline Al ( $\mu \mathrm{g} / \mathrm{g}$ dry Wt.) & $13.58 \pm 1.378$ & $0-31.06$ & 3.20 & $1.36 \times 10^{-3}$ \\
\hline Ba ( $\mu \mathrm{g} / \mathrm{g}$ dry Wt.) & $1.3 \pm 0.18$ & $0-4.2$ & 0.31 & $1.3 \times 10^{-4}$ \\
\hline Co ( $\mu \mathrm{g} / \mathrm{g}$ dry Wt.) & <D.L. & ----- & ----- & ------- \\
\hline $\mathrm{Cr}$ ( $\mu \mathrm{g} / \mathrm{g}$ dry Wt.) & $0.25 \pm 0.01$ & $0.08-0.48$ & 0.06 & $2.5 \times 10^{-5}$ \\
\hline $\mathrm{Cu}(\mu \mathrm{g} / \mathrm{g}$ dry Wt.) & $14.28 \pm 0.14$ & $12.14-15.96$ & 3.43 & $1.43 \times 10^{-3}$ \\
\hline $\operatorname{Er}(\mu \mathrm{g} / g$ dry Wt.) & <D.L. & ------ & ------ & ------- \\
\hline Fe ( $\mu \mathrm{g} / \mathrm{g}$ dry Wt.) & $275.82 \pm 2.6$ & 254.39-305.9 & 66.18 & $2.76 \times 10^{-2}$ \\
\hline Mn ( $\mu \mathrm{g} / \mathrm{g}$ dry Wt.) & $1.4 \pm 0.03$ & $1.13-1.25$ & 0.34 & $1.4 \times 10^{-4}$ \\
\hline Mo ( $\mu \mathrm{g} / \mathrm{g}$ dry Wt.) & <D.L. & ------ & ------ & ------- \\
\hline $\mathrm{Ni}$ ( $\mu \mathrm{g} / \mathrm{g}$ dry Wt.) & < D.L. & ------ & ------ & ------- \\
\hline $\mathrm{Pb}(\mu \mathrm{g} / \mathrm{g}$ dry Wt.) & < D.L. & ------ & ------ & ------ \\
\hline $\mathrm{Sb}(\mu \mathrm{g} / \mathrm{g}$ dry Wt.) & < D.L. & ------ & ------ & ------- \\
\hline Se $(\mu \mathrm{g} / \mathrm{g}$ dry $\mathrm{Wt})$. & $1.49 \pm 0.05$ & $0.83-2.15$ & 0.36 & $1.49 \times 10^{-4}$ \\
\hline Si ( $\mu \mathrm{g} / \mathrm{g}$ dry Wt.) & $31.34 \pm 1.03$ & $21.43-47$ & 5.10 & $3.13 \times 10^{-3}$ \\
\hline $\mathrm{Sr}(\mu \mathrm{g} / \mathrm{g}$ dry Wt.) & $1.74 \pm 0.06$ & $0.18-6.99$ & 0.46 & $1.74 \times 10^{-4}$ \\
\hline Th ( $\mu \mathrm{g} / \mathrm{g}$ dry Wt.) & <D.L. & ------ & ------ & ------- \\
\hline Ti ( $\mu g / g$ dry Wt.) & < D.L. & ------ & ------ & ------- \\
\hline TI ( $\mu \mathrm{g} / \mathrm{g}$ dry Wt.) & < D.L. & ------ & ------ & ------- \\
\hline Tm ( $\mu \mathrm{g} / \mathrm{g}$ dry Wt.) & < D.L. & ------- & ------- & ------- \\
\hline
\end{tabular}

Arab J. Nucl. Sci. \& Applic. Vol. 53, No.3 (2020) 
INVESTIGATION OF SOME ELEMENTS CONCENTRATIONS...

\begin{tabular}{|c|c|c|c|c|}
\hline $\mathrm{W}$ ( $\mu \mathrm{g} / \mathrm{g}$ dry Wt.) & $1.74 \pm 0.06$ & $1.16-2.81$ & 0.42 & $1.74 \times 10^{-4}$ \\
\hline Zn ( $\mu \mathrm{g} / \mathrm{g}$ dry Wt.) & $83.99 \pm 1.14$ & $68.8-100.60$ & 20.15 & $8.4 \times 10^{-3}$ \\
\hline $\mathrm{Zr}(\mu \mathrm{g} / \mathrm{g}$ dry Wt.) & <D.L. & ------ & ------ & ------- \\
\hline
\end{tabular}

Table (9): mean concentrations, ranges and Abundances of detected Elements in $C$. japonica Intestines

\begin{tabular}{ccccc}
\hline Element & Mean $(\mu \mathrm{g} / \mathrm{g}$ dry Wt. $) \pm$ SD & Range $(\mu \mathrm{g} / \mathrm{g}$ dry Wt. $)$ & Abundance $(\%)$ & Concentration \% w/w \\
\hline $\mathrm{Al}(\mu \mathrm{g} / \mathrm{g}$ dry Wt. $)$ & $101.88 \pm 5.97$ & $22.92-168.02$ & 101.9 & $1.02 \times 10^{-2}$ \\
$\mathrm{Ba}(\mu \mathrm{g} / \mathrm{g}$ dry Wt. & $6.76 \pm 0.31$ & $2.34-9.99$ & 6.8 & $6.76 \times 10^{-4}$ \\
$\mathrm{Co}(\mu \mathrm{g} / \mathrm{g}$ dry Wt. $)$ & $0.7 \pm 0.01$ & $0.53-0.87$ & 0.7 & $7 \times 10^{-5}$ \\
$\mathrm{Cr}(\mu \mathrm{g} / \mathrm{g}$ dry Wt. $)$ & $7.92 \pm 0.67$ & $1.92-15.48$ & 7.9 & $7.92 \times 10^{-4}$ \\
$\mathrm{Cu}(\mu \mathrm{g} / \mathrm{g}$ dry Wt. $)$ & $17 \pm 0.67$ & $11.23-27.7$ & 17.0 & $1.7 \times 10^{-3}$ \\
$\mathrm{Er}(\mu \mathrm{g} / \mathrm{g}$ dry Wt. & $1.41 \pm 0.18$ & $0-4.05$ & 1.4 & $1.41 \times 10^{-4}$ \\
$\mathrm{Fe}(\mu \mathrm{g} / \mathrm{g}$ dry Wt. $)$ & $210.3 \pm 6.64$ & $142.01-281.23$ & 210.3 & $2.1 \times 10^{-2}$ \\
$\mathrm{Mn}(\mu \mathrm{g} / \mathrm{g}$ dry Wt. $)$ & $59.07 \pm 1.99$ & $32.13-79.48$ & 59.1 & $5.91 \times 10^{-3}$ \\
\hline
\end{tabular}

Table (9) continued: mean concentrations, ranges and Abundances of detected Elements in $C$. japonica Intestines

\begin{tabular}{|c|c|c|c|c|}
\hline Element & $\operatorname{Mean}(\mu \mathrm{g} / \mathrm{g}$ dry Wt. $) \pm \mathrm{SD}$ & Range( $\mu \mathrm{g} / \mathrm{g}$ dry Wt. $)$ & Abundance (\%) & Concentration $\% \mathrm{w} / \mathrm{w}$ \\
\hline Mo $(\mu \mathrm{g} / \mathrm{g}$ dry Wt. $)$ & $<$ D.L. & ------ & ------ & ------- \\
\hline Ni ( $\mu$ g/g dry Wt.) & $3.39 \pm 0.09$ & $2.32-4.51$ & 3.4 & $3.39 \times 10^{-4}$ \\
\hline $\mathrm{Pb}(\mu \mathrm{g} / \mathrm{g}$ dry Wt. $)$ & < D.L. & ------ & ------ & ------- \\
\hline $\mathrm{Sb}(\mu \mathrm{g} / \mathrm{g}$ dry Wt. $)$ & $0.55 \pm 0.05$ & $0-1.3$ & 0.08 & $5.5 \times 10^{-5}$ \\
\hline Se $(\mu \mathrm{g} / \mathrm{g}$ dry Wt. $)$ & $0.88 \pm 0.04$ & $0.38-1.49$ & 0.13 & $8.8 \times 10^{-5}$ \\
\hline Si ( $\mu \mathrm{g} / \mathrm{g}$ dry Wt.) & $135.84 \pm 8.98$ & $51.45-255.39$ & 19.39 & $1.36 \times 10^{-2}$ \\
\hline $\mathrm{Sr}(\mu \mathrm{g} / \mathrm{g}$ dry Wt. $)$ & $26.97 \pm 0.71$ & $21.83-38.89$ & 3.85 & $2.7 \times 10^{-3}$ \\
\hline Th ( $\mu \mathrm{g} / \mathrm{g}$ dry Wt.) & $1.23 \pm 0.04$ & $0.744-1.86$ & 0.18 & $1.23 \times 10^{-4}$ \\
\hline $\mathrm{Ti}(\mu \mathrm{g} / \mathrm{g}$ dry Wt. $)$ & < D.L. & ------ & ------ & ------- \\
\hline $\mathrm{Tl}(\mu \mathrm{g} / \mathrm{g}$ dry Wt. $)$ & $<$ D.L. & ------ & ------ & ------- \\
\hline Tm ( $\mu \mathrm{g} / \mathrm{g}$ dry Wt. $)$ & < D.L. & ------ & ------ & ------- \\
\hline $\mathrm{W}(\mu \mathrm{g} / \mathrm{g}$ dry Wt. $)$ & $2.54 \pm 0.05$ & $1.89-3.03$ & 0.36 & $2.54 \times 10^{-4}$ \\
\hline $\mathrm{Zn}(\mu \mathrm{g} / \mathrm{g}$ dry Wt. $)$ & $124.27 \pm 2.25$ & $90.26-153.15$ & 17.73 & $1.24 \times 10^{-2}$ \\
\hline $\mathrm{Zr}(\mu \mathrm{g} / \mathrm{g}$ dry Wt. $)$ & < D.L. & ------ & ------ & ------- \\
\hline
\end{tabular}

Table (10): mean concentrations, ranges and Abundances of detected Elements in $C$. japonica Kidneys

\begin{tabular}{|c|c|c|c|c|}
\hline Element & Mean $(\mu \mathrm{g} / \mathrm{g}$ dry Wt. $) \pm \mathrm{SD}$ & Range $(\mu \mathrm{g} / \mathrm{g}$ dry Wt. $)$ & Abundance (\%) & Concentration $\% \mathrm{w} / \mathrm{w}$ \\
\hline Al ( $\mu \mathrm{g} / \mathrm{g}$ dry Wt.) & $6.81 \pm 0.81$ & $0-18.23$ & 1.63 & $6.81 \times 10^{-4}$ \\
\hline $\mathrm{Ba}(\mu \mathrm{g} / \mathrm{g}$ dry $\mathrm{Wt})$. & < D.L. & ----- & ------ & ------- \\
\hline Co ( $\mu \mathrm{g} / \mathrm{g}$ dry Wt.) & < D.L. & ------ & ----- & ------- \\
\hline $\mathrm{Cr}(\mu \mathrm{g} / \mathrm{g}$ dry Wt.) & $0.29 \pm 0.01$ & $0.22-0.49$ & 0.07 & $2.9 \times 10^{-5}$ \\
\hline $\mathrm{Cu}(\mu \mathrm{g} / \mathrm{g}$ dry Wt.) & $12.41 \pm 0.05$ & $11.93-13.04$ & 3.06 & $1.24 \times 10^{-3}$ \\
\hline $\operatorname{Er}(\mu \mathrm{g} / \mathrm{g}$ dry $W \mathrm{t})$. & <D.L. & ------ & ------ & ------- \\
\hline Fe ( $\mu \mathrm{g} / \mathrm{g}$ dry Wt.) & $267.89 \pm 4.49$ & $206.32-327.25$ & 66.04 & $2.68 \times 10^{-2}$ \\
\hline $\mathrm{Mn}(\mu \mathrm{g} / \mathrm{g}$ dry Wt.) & $7.91 \pm 0.28$ & 5.34-11.8 & 1.95 & $7.91 \times 10^{-4}$ \\
\hline Mo ( $\mu \mathrm{g} / \mathrm{g}$ dry Wt.) & $3.83 \pm 0.04$ & $3.5-4.43$ & 0.94 & $3.83 \times 10^{-4}$ \\
\hline $\mathrm{Ni}(\mu \mathrm{g} / \mathrm{g}$ dry Wt.) & <D.L. & ------ & ------ & ------- \\
\hline $\mathrm{Pb}(\mu \mathrm{g} / \mathrm{g}$ dry Wt.) & <D.L. & ------ & ------ & ------- \\
\hline $\mathrm{Sb}(\mu \mathrm{g} / \mathrm{g}$ dry $\mathrm{Wt})$. & $0.21 \pm 0.01$ & $0-0.3$ & 0.05 & $2.1 \times 10^{-5}$ \\
\hline Se ( $\mu \mathrm{g} / \mathrm{g}$ dry Wt.) & $4.47 \pm 0.06$ & $3.88-5.3$ & 1.10 & $4.47 \times 10^{-4}$ \\
\hline Si ( $\mu \mathrm{g} / \mathrm{g}$ dry Wt.) & $24.8 \pm 1.8$ & $10.68-51.43$ & 6.11 & $2.48 \times 10^{-3}$ \\
\hline $\operatorname{Sr}(\mu \mathrm{g} / \mathrm{g}$ dry Wt.) & <D.L. & ------ & ------ & ------- \\
\hline Th ( $\mu \mathrm{g} / \mathrm{g}$ dry $\mathrm{Wt})$. & $0.22 \pm 0.02$ & $0-0.4$ & 0.06 & $2.2 \times 10^{-5}$ \\
\hline Ti ( $\mu \mathrm{g} / \mathrm{g}$ dry Wt.) & <D.L. & ------ & ----- & ------- \\
\hline Tl ( $\mu \mathrm{g} / \mathrm{g}$ dry Wt.) & < D.L. & ----- & ----- & ------- \\
\hline Tm ( $\mu \mathrm{g} / \mathrm{g}$ dry Wt.) & <D.L. & ------ & ------ & ------- \\
\hline W ( $\mu \mathrm{g} / \mathrm{g}$ dry Wt.) & $1.17 \pm 0.02$ & 0.96-1.46 & 0.29 & $1.17 \times 10^{-4}$ \\
\hline Zn ( $\mu \mathrm{g} / \mathrm{g}$ dry Wt.) & $75.82 \pm 2.3$ & $62.07-110.17$ & 18.69 & $7.58 \times 10-3$ \\
\hline 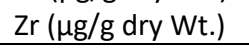 & <D.L. & ------ & ------ & ------- \\
\hline
\end{tabular}


Table (11): mean concentrations, ranges and Abundances of detected Elements in $C$. japonica Lungs

\begin{tabular}{|c|c|c|c|c|}
\hline Element & $\operatorname{Mean}(\mu \mathrm{g} / \mathrm{g}$ dry Wt. $) \pm \mathrm{SD}$ & Range $(\mu \mathrm{g} / \mathrm{g}$ dry Wt. $)$ & Abundance $(\%)$ & Concentration $\% \mathrm{w} / \mathrm{w}$ \\
\hline $\mathrm{Al}(\mu \mathrm{g} / \mathrm{g}$ dry Wt. $)$ & $52.34 \pm 4.8$ & $12-105.5$ & 6.06 & $5.23 \times 10^{-3}$ \\
\hline $\mathrm{Ba}(\mu \mathrm{g} / \mathrm{g}$ dry $\mathrm{Wt})$. & < D.L. & ------ & ------ & ------- \\
\hline Co $(\mu \mathrm{g} / \mathrm{g}$ dry Wt. $)$ & < D.L. & ------ & ------ & ------- \\
\hline $\mathrm{Cr}(\mu \mathrm{g} / \mathrm{g}$ dry $\mathrm{Wt})$. & $0.38 \pm 0.03$ & $0.19-0.74$ & 0.04 & $3.8 \times 10^{-5}$ \\
\hline $\mathrm{Cu}(\mu \mathrm{g} / \mathrm{g}$ dry $\mathrm{Wt})$. & $3.17 \pm 0.13$ & $2.04-4.55$ & 0.37 & $3.17 \times 10^{-4}$ \\
\hline Er $(\mu \mathrm{g} / \mathrm{g}$ dry Wt. $)$ & $0.89 \pm 0.08$ & $0.38-1.87$ & 0.10 & $8.9 \times 10^{-5}$ \\
\hline $\mathrm{Fe}(\mu \mathrm{g} / \mathrm{g}$ dry Wt. $)$ & $676.83 \pm 18.64$ & $545.06-808.6$ & 78.35 & $6.78 \times 10^{-2}$ \\
\hline $\mathrm{Mn}(\mu \mathrm{g} / \mathrm{g}$ dry Wt. $)$ & $3.3 \pm 0.10$ & $2.54-4.45$ & 0.38 & $3.3 \times 10^{-4}$ \\
\hline Mo ( $\mu \mathrm{g} / \mathrm{g}$ dry Wt.) & < D.L. & ------ & ------ & ------- \\
\hline $\mathrm{Ni}(\mu \mathrm{g} / \mathrm{g}$ dry Wt. & < D.L. & ------ & ------ & ------- \\
\hline $\mathrm{Pb}(\mu \mathrm{g} / \mathrm{g}$ dry $\mathrm{Wt})$. & < D.L. & ------ & ------ & ------- \\
\hline $\mathrm{Sb}(\mu \mathrm{g} / \mathrm{g}$ dry $\mathrm{Wt})$. & $<$ D.L. & ------ & ----- & ------- \\
\hline $\mathrm{Se}(\mu \mathrm{g} / \mathrm{g}$ dry Wt. $)$ & $0.92 \pm 0.06$ & $0.32-1.46$ & 0.11 & $9.2 \times 10^{-5}$ \\
\hline Si ( $\mu \mathrm{g} / \mathrm{g}$ dry Wt.) & $44.22 \pm 2.5$ & 27.59-73.09 & 5.12 & $4.42 \times 10^{-3}$ \\
\hline $\mathrm{Sr}(\mu \mathrm{g} / \mathrm{g}$ dry Wt. $)$ & < D.L. & ------ & ------ & ------- \\
\hline Th ( $\mu \mathrm{g} / \mathrm{g}$ dry Wt.) & $0.75 \pm 0.025$ & $0-0.84$ & 0.09 & $2.2 \times 10^{-5}$ \\
\hline Ti ( $\mu \mathrm{g} / \mathrm{g}$ dry Wt.) & < D.L. & ------ & ----- & ------- \\
\hline $\mathrm{Tl}(\mu \mathrm{g} / \mathrm{g}$ dry Wt. $)$ & < D.L. & ------ & ------ & ------- \\
\hline $\operatorname{Tm}(\mu \mathrm{g} / \mathrm{g}$ dry Wt. $)$ & $<$ D.L. & ------ & ----- & ------- \\
\hline $\mathrm{W}(\mu \mathrm{g} / \mathrm{g}$ dry Wt. $)$ & $0.99 \pm 0.06$ & $0.55-1.69$ & 0.11 & $9.9 \times 10^{-5}$ \\
\hline $\mathrm{Zn}(\mu \mathrm{g} / \mathrm{g}$ dry Wt. $)$ & $80.09 \pm 5$ & $30.06-129.6$ & 9.27 & $8.01 \times 10^{-3}$ \\
\hline $\mathrm{Zr}(\mu \mathrm{g} / \mathrm{g}$ dry $\mathrm{Wt})$. & < D.L. & ------ & ------ & ------- \\
\hline
\end{tabular}

Table (12): mean concentrations, ranges and Abundances of detected Elements in $C$. japonica Liver

\begin{tabular}{|c|c|c|c|c|}
\hline Element & $\operatorname{Mean}(\mu \mathrm{g} / \mathrm{g}$ dry Wt. $) \pm \mathrm{SD}$ & Range $(\mu \mathrm{g} / \mathrm{g}$ dry Wt.) & Abundance (\%) & Concentration $\% \mathrm{w} / \mathrm{w}$ \\
\hline $\mathrm{Al}(\mu \mathrm{g} / \mathrm{g}$ dry Wt.) & $1.88 \pm 0.29$ & $0-6.91$ & 0.49 & $1.88 \times 10^{-4}$ \\
\hline $\mathrm{Ba}(\mu \mathrm{g} / \mathrm{g}$ dry $\mathrm{Wt})$. & < D.L. & ------ & ------ & ------- \\
\hline Co $(\mu \mathrm{g} / \mathrm{g}$ dry Wt. $)$ & < D.L. & ------ & ------ & ------- \\
\hline $\mathrm{Cr}(\mu \mathrm{g} / \mathrm{g}$ dry $\mathrm{Wt})$. & $0.22 \pm 0.01$ & $0.12-0.32$ & 0.06 & $0.22 \times 10^{-5}$ \\
\hline $\mathrm{Cu}(\mu \mathrm{g} / \mathrm{g}$ dry Wt. $)$ & $12.24 \pm 0.15$ & $10.68-14.81$ & 3.19 & $1.22 \times 10^{-3}$ \\
\hline $\operatorname{Er}(\mu \mathrm{g} / \mathrm{g}$ dry $\mathrm{Wt})$. & $0.06 \pm 0.007$ & $0-0.17$ & 0.01 & $6 \times 10^{-6}$ \\
\hline $\mathrm{Fe}(\mu \mathrm{g} / \mathrm{g}$ dry Wt. $)$ & $251.67 \pm 1.9$ & $228.24-270.51$ & 65.64 & $2.51 \times 10^{-2}$ \\
\hline $\mathrm{Mg}(\mu \mathrm{g} / \mathrm{g}$ dry Wt. $)$ & $243.31 \pm 5.11$ & 199.83-315.95 & 0.41 & $2.43 \times 10^{-2}$ \\
\hline Mn $(\mu \mathrm{g} / \mathrm{g}$ dry Wt. $)$ & $7.85 \pm 0.07$ & 6.83-8.71 & 2.05 & $7.85 \times 10^{-4}$ \\
\hline Mo ( $\mu \mathrm{g} / \mathrm{g}$ dry Wt.) & $1.59 \pm 0.02$ & $1.4-1.73$ & 0.01 & $1.59 \times 10^{-4}$ \\
\hline Ni ( $\mu \mathrm{g} / \mathrm{g}$ dry Wt.) & < D.L. & ------ & ------ & ------- \\
\hline $\mathrm{Pb}(\mu \mathrm{g} / \mathrm{g}$ dry $\mathrm{Wt})$. & $1.8 \pm 0.37$ & $0-8.39$ & 0.47 & $1.8 \times 10^{-4}$ \\
\hline
\end{tabular}

Table (12) continued: mean concentrations, ranges and Abundances of detected Elements in $C$. japonica Liver

\begin{tabular}{|c|c|c|c|c|}
\hline Element & Mean $(\mu \mathrm{g} / \mathrm{g}$ dry Wt. $) \pm \mathrm{SD}$ & Range( $\mu \mathrm{g} / \mathrm{g}$ dry Wt. $)$ & Abundance (\%) & Concentration $\% \mathrm{w} / \mathrm{w}$ \\
\hline $\mathrm{Sb}(\mu \mathrm{g} / \mathrm{g}$ dry Wt. $)$ & $0.45 \pm 0.01$ & $0.3-0.56$ & 0.12 & $4.5 \times 10^{-5}$ \\
\hline Se ( $\mu \mathrm{g} / \mathrm{g}$ dry $\mathrm{Wt})$. & $2.26 \pm 0.04$ & $1.8-2.69$ & 0.59 & $2.26 \times 10^{-4}$ \\
\hline $\mathrm{Si}(\mu \mathrm{g} / \mathrm{g}$ dry Wt.) & $14.8 \pm 0.79$ & $7.25-27.76$ & 3.86 & $1.48 \times 10^{-3}$ \\
\hline $\mathrm{Sr}(\mu \mathrm{g} / \mathrm{g}$ dry Wt. $)$ & <D.L. & ------ & ------ & ------- \\
\hline Th $(\mu \mathrm{g} / \mathrm{g}$ dry Wt. $)$ & $0.12 \pm 0.01$ & $0.0 .07-0.19$ & 0.03 & $1.2 \times 10^{-5}$ \\
\hline $\mathrm{Ti}(\mu \mathrm{g} / \mathrm{g}$ dry Wt.) & < D.L. & ------ & ----- & ------- \\
\hline $\mathrm{Tl}(\mu \mathrm{g} / \mathrm{g}$ dry Wt.) & $<$ D.L. & ----- & ------ & ------- \\
\hline Tm $(\mu \mathrm{g} / \mathrm{g}$ dry Wt. $)$ & $<$ D.L. & ------ & ------ & ------- \\
\hline $\mathrm{W}(\mu \mathrm{g} / \mathrm{g}$ dry Wt.) & $1.3 \pm 0.05$ & $0.7-2$ & 0.34 & $1.3 \times 10^{-4}$ \\
\hline $\mathrm{Zn}(\mu \mathrm{g} / \mathrm{g}$ dry Wt. $)$ & $87.16 \pm 2.86$ & $53.21-126.95$ & 22.73 & $8.71 \times 10^{-3}$ \\
\hline $\mathrm{Zr}(\mu \mathrm{g} / \mathrm{g}$ dry $\mathrm{Wt})$. & <D.L. & ------ & ------ & ------- \\
\hline
\end{tabular}


Table (13): mean concentrations, ranges and Abundances of detected Elements in $C$. japonica Ovaries

\begin{tabular}{|c|c|c|c|c|}
\hline Element & Mean $(\mu \mathrm{g} / \mathrm{g}$ dry Wt. $) \pm \mathrm{SD}$ & Range ( $\mu \mathrm{g} / \mathrm{g}$ dry Wt.) & Abundance (\%) & Concentration $\% \mathrm{w} / \mathrm{w}$ \\
\hline $\mathrm{Al}(\mu \mathrm{g} / \mathrm{g}$ dry Wt.) & $<$ D.L. & ----- & ------ & ------- \\
\hline $\mathrm{Ba}(\mu \mathrm{g} / \mathrm{g}$ dry Wt. $)$ & < D.L. & ------ & ------ & ------- \\
\hline Co $(\mu \mathrm{g} / \mathrm{g}$ dry Wt. $)$ & $<$ D.L. & ------ & ------ & ------- \\
\hline $\mathrm{Cr}(\mu \mathrm{g} / \mathrm{g}$ dry Wt. $)$ & $0.14 \pm 0.01$ & $0.11-0.17$ & 0.13 & $1.4 \times 10^{-5}$ \\
\hline $\mathrm{Cu}(\mu \mathrm{g} / \mathrm{g}$ dry $\mathrm{Wt})$. & $4.25 \pm 0.28$ & $2.59-5.61$ & 3.94 & $4.25 \times 10^{-4}$ \\
\hline $\operatorname{Er}(\mu \mathrm{g} / \mathrm{g}$ dry Wt. $)$ & < D.L. & ----- & ----- & ------ \\
\hline $\mathrm{Fe}(\mu \mathrm{g} / \mathrm{g}$ dry Wt. $)$ & $35.52 \pm 2.37$ & $30.25-39.65$ & 32.98 & $3.55 \times 10^{-3}$ \\
\hline Mn ( $\mu \mathrm{g} / \mathrm{g}$ dry Wt. $)$ & $1.28 \pm 0.09$ & $0.96-1.57$ & 1.19 & $1.28 \times 10^{-4}$ \\
\hline Mo ( $\mu \mathrm{g} / \mathrm{g}$ dry Wt.) & < D.L. & ------ & $-\cdots$ & ------ \\
\hline $\mathrm{Ni}(\mu \mathrm{g} / \mathrm{g}$ dry Wt.) & $2.32 \pm 0.16$ & $1.62-3.11$ & 2.18 & $2.32 \times 10^{-4}$ \\
\hline $\mathrm{Pb}(\mu \mathrm{g} / \mathrm{g}$ dry Wt. $)$ & < D.L. & ------ & ------ & ------- \\
\hline $\mathrm{Sb}(\mu \mathrm{g} / \mathrm{g}$ dry Wt. $)$ & $0.2 \pm 0.01$ & $0.13-0.35$ & 0.19 & $2 \times 10^{-5}$ \\
\hline Se $(\mu \mathrm{g} / \mathrm{g}$ dry Wt. $)$ & $1.33 \pm 0.09$ & $1.1-1.54$ & 1.23 & $7.9 \times 10^{-5}$ \\
\hline Si $(\mu \mathrm{g} / \mathrm{g}$ dry Wt. $)$ & $9.7 \pm 0.65$ & $5.21-12.65$ & 9.01 & $9.7 \times 10^{-4}$ \\
\hline $\mathrm{Sr}(\mu \mathrm{g} / \mathrm{g}$ dry $\mathrm{Wt})$. & < D.L. & ------ & ------ & ------- \\
\hline Th $(\mu \mathrm{g} / \mathrm{g}$ dry Wt. $)$ & $<$ D.L. & ------ & ------ & ------- \\
\hline Ti ( $\mu \mathrm{g} / \mathrm{g}$ dry Wt.) & $<$ D.L. & ------ & ------ & ------- \\
\hline $\mathrm{Tl}(\mu \mathrm{g} / \mathrm{g}$ dry Wt. $)$ & $<$ D.L. & ------ & ----- & ------- \\
\hline Tm ( $\mu \mathrm{g} / \mathrm{g}$ dry Wt. $)$ & <D.L. & ------ & ------ & ------- \\
\hline $\mathrm{W}(\mu \mathrm{g} / \mathrm{g}$ dry Wt.) & $0.96 \pm 0.06$ & $0.4-1.2$ & 0.89 & $9.6 \times 10^{-5}$ \\
\hline $\mathrm{Zn}(\mu \mathrm{g} / \mathrm{g}$ dry Wt. $)$ & $52 \pm 3.47$ & $42.51-55.68$ & 48.28 & $5.2 \times 10^{-3}$ \\
\hline $\mathrm{Zr}(\mu \mathrm{g} / \mathrm{g}$ dry Wt. $)$ & <D.L. & ------ & ------ & ------- \\
\hline
\end{tabular}

Table (14): mean concentrations, ranges and Abundances of detected Elements in $C$. japonica Skeletal Muscles

\begin{tabular}{|c|c|c|c|c|}
\hline Element & Mean $(\mu \mathrm{g} / \mathrm{g}$ dry Wt. $) \pm \mathrm{SD}$ & Range $(\mu \mathrm{g} / \mathrm{g}$ dry Wt. $)$ & Abundance $(\%)$ & Concentration $\% \mathrm{w} / \mathrm{w}$ \\
\hline $\mathrm{Al}(\mu \mathrm{g} / \mathrm{g}$ dry $\mathrm{Wt})$. & $<$ D.L. & ----- & ------ & ------- \\
\hline $\mathrm{Ba}(\mu \mathrm{g} / \mathrm{g}$ dry Wt.) & $<$ D.L. & ------ & ------ & ------- \\
\hline Co ( $\mu \mathrm{g} / \mathrm{g}$ dry Wt. $)$ & $<$ D.L. & ------ & ------ & ------- \\
\hline $\mathrm{Cr}(\mu \mathrm{g} / \mathrm{g}$ dry Wt. $)$ & < D.L. & ------ & ------ & ------- \\
\hline $\mathrm{Cu}(\mu \mathrm{g} / \mathrm{g}$ dry Wt. $)$ & $0.45 \pm 0.01$ & $0.38-0.59$ & 6.78 & $4.5 \times 10^{-5}$ \\
\hline $\operatorname{Er}(\mu \mathrm{g} / \mathrm{g}$ dry Wt. $)$ & <D.L. & ------ & ------ & ------- \\
\hline $\mathrm{Fe}(\mu \mathrm{g} / \mathrm{g}$ dry $\mathrm{Wt})$. & $5.81 \pm 0.08$ & $3.62-8.76$ & 86.69 & $5.81 \times 10^{-4}$ \\
\hline Mn $(\mu \mathrm{g} / \mathrm{g}$ dry Wt. $)$ & < D.L. & ------ & ------ & ------- \\
\hline Mo $(\mu \mathrm{g} / \mathrm{g}$ dry Wt. $)$ & $<$ D.L. & ------ & ----- & ------- \\
\hline $\mathrm{Ni}(\mu \mathrm{g} / \mathrm{g}$ dry Wt. & $<$ D.L. & ------ & ------ & ------- \\
\hline $\mathrm{Pb}(\mu \mathrm{g} / \mathrm{g}$ dry Wt. $)$ & < D.L. & ------ & ------ & ------- \\
\hline $\mathrm{Sb}(\mu \mathrm{g} / \mathrm{g}$ dry Wt. $)$ & < D.L. & ------ & ------ & ------- \\
\hline Se ( $\mu \mathrm{g} / \mathrm{g}$ dry Wt.) & $0.79 \pm 0.01$ & $0.24-0.87$ & 11.8 & $7.9 \times 10^{-5}$ \\
\hline Si ( $\mu \mathrm{g} / \mathrm{g}$ dry Wt.) & $1.48 \pm 0.01$ & $0.86-2.16$ & 22.16 & $1.48 \times 10^{-4}$ \\
\hline $\mathrm{Sr}(\mu \mathrm{g} / \mathrm{g}$ dry $\mathrm{Wt})$. & < D.L. & ------ & ------ & ------- \\
\hline Th $(\mu \mathrm{g} / \mathrm{g}$ dry Wt. $)$ & $<$ D.L. & ------ & ------ & ------- \\
\hline $\mathrm{Ti}(\mu \mathrm{g} / \mathrm{g}$ dry Wt.) & < D.L. & ------ & ------ & ------- \\
\hline $\mathrm{Tl}(\mu \mathrm{g} / \mathrm{g}$ dry Wt. $)$ & $0.41 \pm 0.01$ & $0.0 .32-0.52$ & 6.13 & $0.41 \times 10^{-5}$ \\
\hline Tm $(\mu \mathrm{g} / \mathrm{g}$ dry Wt. $)$ & < D.L. & ----- & ----- & ------- \\
\hline $\mathrm{W}(\mu \mathrm{g} / \mathrm{g}$ dry $\mathrm{Wt})$. & $<$ D.L. & ------ & ----- & ------- \\
\hline $\mathrm{Zn}(\mu \mathrm{g} / \mathrm{g}$ dry Wt.) & $2.8 \pm 0.06$ & $0.66-1.28$ & 41.87 & $2.8 \times 10^{-4}$ \\
\hline $\mathrm{Zr}(\mu \mathrm{g} / \mathrm{g}$ dry $\mathrm{Wt})$. & $1.21 \pm 0.08$ & $0.42-1.97$ & 18.03 & $1.21 \times 10^{-5}$ \\
\hline
\end{tabular}


Table (15): mean concentrations, ranges and Abundances of detected Elements in $C$. japonica Coagulated Blood

\begin{tabular}{|c|c|c|c|c|}
\hline Element & Mean $(\mu \mathrm{g} / \mathrm{g}$ dry Wt. $) \pm \mathrm{SD}$ & $\operatorname{Range}(\mu \mathrm{g} / \mathrm{g}$ dry Wt. $)$ & Abundance (\%) & Concentration $\% \mathrm{w} / \mathrm{w}$ \\
\hline $\mathrm{Al}(\mu \mathrm{g} / \mathrm{g}$ dry Wt.) & < D.L. & ----- & ----- & ------- \\
\hline $\mathrm{Ba}(\mu \mathrm{g} / \mathrm{g}$ dry Wt. $)$ & $<$ D.L. & ------ & ------ & ------- \\
\hline Co $(\mu \mathrm{g} / \mathrm{g}$ dry Wt. $)$ & $<$ D.L. & ------ & ------ & ------- \\
\hline $\mathrm{Cr}(\mu \mathrm{g} / \mathrm{g}$ dry Wt. $)$ & < D.L. & ------ & ------ & ------- \\
\hline $\mathrm{Cu}(\mu \mathrm{g} / \mathrm{g}$ dry Wt. $)$ & $0.76 \pm 0.02$ & $0.6-1.02$ & 0.26 & $7.6 \times 10^{-5}$ \\
\hline $\mathrm{Er}(\mu \mathrm{g} / \mathrm{g}$ dry Wt. $)$ & < D.L. & ------ & ------ & ------- \\
\hline $\mathrm{Fe}(\mu \mathrm{g} / \mathrm{g}$ dry Wt. $)$ & $275.64 \pm 4.8$ & 223.47-321.49 & 95.50 & $2.76 \times 10^{-2}$ \\
\hline $\mathrm{Mn}(\mu \mathrm{g} / \mathrm{g}$ dry Wt. $)$ & < D.L. & ------ & ------ & ------- \\
\hline Mo ( $\mu \mathrm{g} / \mathrm{g}$ dry Wt. & $<$ D.L. & ------ & ------ & ------- \\
\hline Ni ( $\mu$ g/g dry Wt. $)$ & $<$ D.L. & ------ & ------ & ------- \\
\hline $\mathrm{Pb}(\mu \mathrm{g} / \mathrm{g}$ dry Wt. $)$ & $0.5 \pm 0.04$ & $0.18-0.98$ & 0.17 & $5 \times 10^{-5}$ \\
\hline $\mathrm{Sb}(\mu \mathrm{g} / \mathrm{g}$ dry $\mathrm{Wt})$. & < D.L. & ------ & ------ & ------- \\
\hline Se $(\mu \mathrm{g} / \mathrm{g}$ dry Wt. $)$ & $0.62 \pm 0.01$ & $0.54-0.73$ & 0.21 & $5.4 \times 10^{-5}$ \\
\hline
\end{tabular}

Table (15) continued: mean concentrations, ranges and Abundances of detected Elements in $C$. japonica Coagulated

\begin{tabular}{|c|c|c|c|c|}
\hline & Blood & & & \\
\hline Element & $\operatorname{Mean}(\mu \mathrm{g} / \mathrm{g}$ dry Wt. $) \pm \mathrm{SD}$ & Range $(\mu \mathrm{g} / \mathrm{g}$ dry Wt. $)$ & Abundance (\%) & Concentration $\% \mathrm{w} / \mathrm{w}$ \\
\hline $\mathrm{Si}(\mu \mathrm{g} / \mathrm{g}$ dry Wt.) & $2.16 \pm 0.11$ & $0.89-3.16$ & 0.75 & $2.16 \times 10^{-4}$ \\
\hline $\mathrm{Sr}(\mu \mathrm{g} / \mathrm{g}$ dry $\mathrm{Wt})$. & < D.L. & ------ & ----- & ------- \\
\hline Th ( $\mu \mathrm{g} / \mathrm{g}$ dry Wt.) & $<$ D.L. & ------ & ------ & ------- \\
\hline $\mathrm{Ti}(\mu \mathrm{g} / \mathrm{g}$ dry Wt. & < D.L. & ------ & ------ & ------- \\
\hline $\mathrm{Tl}(\mu \mathrm{g} / \mathrm{g}$ dry Wt. $)$ & $0.91 \pm 0.02$ & $0.7-1.16$ & 0.32 & $9.1 \times 10^{-5}$ \\
\hline Tm ( $\mu \mathrm{g} / \mathrm{g}$ dry Wt. $)$ & < D.L. & ------ & ----- & ------- \\
\hline W ( $\mu \mathrm{g} / \mathrm{g}$ dry Wt.) & $<$ D.L. & ------ & ------ & ------- \\
\hline $\mathrm{Zn}(\mu \mathrm{g} / \mathrm{g}$ dry Wt. $)$ & < D.L. & ------ & ------ & -------- \\
\hline $\mathrm{Zr}(\mu \mathrm{g} / \mathrm{g}$ dry Wt. $)$ & $8.04 \pm 0.23$ & $5.57-10.18$ & 2.79 & $8.04 \times 10^{-4}$ \\
\hline
\end{tabular}

Table (16): mean concentrations, ranges and Abundances of detected Elements in $C$. japonica Blood Serum

\begin{tabular}{|c|c|c|c|c|}
\hline Element & $\operatorname{Mean}(\mu \mathrm{g} / \mathrm{g}$ dry Wt. $) \pm \mathrm{SD}$ & Range $(\mu \mathrm{g} / \mathrm{g}$ dry Wt. $)$ & Abundance (\%) & Concentration $\% \mathrm{w} / \mathrm{w}$ \\
\hline $\mathrm{Al}(\mu \mathrm{g} / \mathrm{g})$ & < D.L. & ----- & ------ & ------- \\
\hline $\mathrm{Ba}(\mu \mathrm{g} / \mathrm{g})$ & <D.L. & ------ & ------ & ------- \\
\hline Co $(\mu \mathrm{g} / \mathrm{g})$ & < D.L. & ------ & ------ & ------- \\
\hline $\mathrm{Cr}(\mu \mathrm{g} / \mathrm{g})$ & < D.L. & ------ & ------ & ------- \\
\hline $\mathrm{Cu}(\mu \mathrm{g} / \mathrm{g})$ & $<$ D.L. & ----- & ------ & ------- \\
\hline $\operatorname{Er}(\mu \mathrm{g} / \mathrm{g})$ & $<$ D.L. & ------ & ------ & ------- \\
\hline $\mathrm{Fe}(\mu \mathrm{g} / \mathrm{g})$ & $10.1 \pm 0.67$ & $2.84-16.12$ & 56.16 & $1.01 \times 10^{-3}$ \\
\hline $\operatorname{Mn}(\mu \mathrm{g} / \mathrm{g})$ & $<$ D.L. & ------ & ------ & ------- \\
\hline Mo $(\mu \mathrm{g} / \mathrm{g})$ & $<$ D.L. & ------ & ------ & ------- \\
\hline $\mathrm{Ni}(\mu \mathrm{g} / \mathrm{g})$ & $<$ D.L. & ------ & ------ & ------- \\
\hline $\mathrm{Pb}(\mu \mathrm{g} / \mathrm{g})$ & $<$ D.L. & ------ & ----- & ------- \\
\hline $\mathrm{Sb}(\mu \mathrm{g} / \mathrm{g})$ & $<$ D.L. & ------ & ------ & ------- \\
\hline $\mathrm{Se}(\mu \mathrm{g} / \mathrm{g})$ & < D.L. & ------ & ------ & ------- \\
\hline $\mathrm{Si}(\mu \mathrm{g} / \mathrm{g})$ & < D.L. & ------ & ----- & ------- \\
\hline $\mathrm{Sr}(\mu \mathrm{g} / \mathrm{g})$ & <D.L. & ------ & ------ & ------- \\
\hline Th $(\mu \mathrm{g} / \mathrm{g})$ & $0.24 \pm 0.01$ & $0-0.31$ & 1.32 & $2.4 \times 10^{-5}$ \\
\hline $\mathrm{Ti}(\mu \mathrm{g} / \mathrm{g})$ & <D.L. & ------ & ------ & ------- \\
\hline $\mathrm{Tl}(\mu \mathrm{g} / \mathrm{g})$ & $0.23 \pm 0.01$ & $0.12-0.36$ & 1.26 & $2.3 \times 10^{-5}$ \\
\hline $\operatorname{Tm}(\mu \mathrm{g} / \mathrm{g})$ & <D.L. & ------ & ------ & ------- \\
\hline $\mathrm{W}(\mu \mathrm{g} / \mathrm{g})$ & $0.3 \pm 0.01$ & $0.18-0.47$ & 1.65 & $3 \times 10^{-5}$ \\
\hline $\mathrm{Zn}(\mu \mathrm{g} / \mathrm{g})$ & $0.92 \pm 0.03$ & $0.66-1.28$ & 5.13 & $9.2 \times 10^{-5}$ \\
\hline $\mathrm{Zr}(\mu \mathrm{g} / \mathrm{g})$ & $6.2 \pm 1.1$ & $0.27-22.74$ & 6.2 & $6.2 \times 10^{-4}$ \\
\hline
\end{tabular}

Arab J. Nucl. Sci. \& Applic. Vol. 53, No.3 (2020) 
Table (17): summarized concentrations of detected Elements in all studied organs of $C$. japonica\#

\begin{tabular}{|c|c|c|c|c|c|c|c|c|c|c|c|c|c|c|c|c|}
\hline Organ & Beak: & Brain & Crop & Eyen & Gizzard & Heast & Intertionet & Kithens & Lang & Livet & Ovarien & BCDS & Serum & Muscle & \multirow{2}{*}{$\begin{array}{l}\text { Element } \\
\text { Total } \\
\text { IIS }\end{array}$} & \multirow{2}{*}{ Elemem } \\
\hline Element & $\begin{array}{l}\text { Ave. } \\
\text { pgig }\end{array}$ & $\begin{array}{l}\text { Ave } \\
\mu g \mathrm{~g}\end{array}$ & $\begin{array}{l}\text { Ave. } \\
\mu g g\end{array}$ & $\begin{array}{l}\text { Ave } \\
\mu g g\end{array}$ & $\begin{array}{l}\text { Ave } \\
\text { ugg }\end{array}$ & $\begin{array}{l}\text { Ave } \\
\mu g g \text {. }\end{array}$ & Ave. $\mu g$ \& & $\begin{array}{l}\text { Aug } \\
\text { Hgg }\end{array}$ & $\begin{array}{l}\text { Alve } \\
\mathrm{egg}\end{array}$ & $\begin{array}{l}\text { Are } \\
\mu g g\end{array}$ & $\begin{array}{l}\text { Ase } \\
\mu g 8\end{array}$ & $\begin{array}{l}\text { Ave } \\
\mu g \mathrm{~B}\end{array}$ & $\begin{array}{l}\text { Are. } \\
\text { Hgg }\end{array}$ & $\begin{array}{l}\text { Ave. } \\
\text { ygg }\end{array}$ & & \\
\hline AI & 36.9 & 163 & 26.6 & 4.8 & 11.72 & 13.4 & 101.9 & 6.6 & 52.3 & 19 & 0.0 & 00 & 00 & 00 & 232.32 & 5,4324 \\
\hline $\mathrm{Bz}$ & 48.6 & 0.0 & 1.4 & 24.4 & 102 & 13 & 68 & 0.0 & 00 & 0.0 & 0.0 & 0.0 & 00 & 0.0 & 8344 & 1.6798 \\
\hline $\mathrm{Co}_{0}$ & 0.0 & 00 & 00 & 0.0 & 0.0 & 0.0 & 6.7 & 0.0 & 00 & 0.0 & 00 & 0.0 & 00 & 00 & 0.70 & 0.0141 \\
\hline$C r$ & 0.6 & 09 & 03 & 0.3 & 0.23 & 0.3 & 79 & 0.3 & 0.4 & 0.2 & 0.1 & 0.0 & 0.0 & 0.0 & 1102 & 0.2359 \\
\hline $\mathrm{Cu}$ & 47 & 120 & 11.0 & 3.0 & 13.97 & 143 & 17.0 & 124 & 32 & 122 & 4.2 & 0.76 & 00 & 0.45 & 10922 & 21989 \\
\hline$E R$ & 10.5 & 15 & 00 & 0.5 & 0.53 & 0.0 & 1.4 & 0.0 & 09 & 01 & 2.0 & 0.0 & 00 & $\infty 0$ & 1531 & 0.3681 \\
\hline $\mathrm{Fe}$ & 200.9 & 1641 & 119.2 & 1472 & 112.49 & 2758 & 2103 & 2679 & 6768 & 251.7 & 35.5 & 27564 & 1011 & 5.51 & 2761.47 & 55.5949 \\
\hline $\mathrm{Ma}$ & 149 & 18 & 35 & 1.8 & 10.29 & 14 & 591 & 79 & 33 & 79 & 13 & 0.0 & 00 & 00 & 11706 & 23567 \\
\hline Mo & 0.0 & 0.0 & 00 & 00 & 0.0 & 0.0 & 0.0 & 3.8 & 20 & 1.6 & 00 & 0.0 & 00 & 0.0 & $54 !$ & 0.1680 \\
\hline $\mathrm{Ni}$ & 00 & 00 & 00 & 0.0 & 0.23 & 0.0 & 3.4 & 0.0 & 20 & 0.0 & 2.3 & 00 & 00 & 0.0 & 593 & 0.1195 \\
\hline$P 6$ & 0.0 & 00 & 00 & 0.0 & 0.0 & 0.0 & 0.0 & 0.0 & 00 & 1.8 & 0.0 & 0.50 & 00 & 00 & 230 & 0.0463 \\
\hline$s t$ & 00 & 0.0 & 00 & 0.0 & 0.0 & 0.0 & 05 & 02 & 00 & 0.5 & 0.2 & 0.0 & 00 & 0.0 & 141 & 0.0284 \\
\hline St & 00 & 00 & 20 & 0.0 & 1.35 & 149 & 09 & 45 & 09 & 23 & 13 & 0.52 & 00 & 0.79 & 1612 & 0.3246 \\
\hline Si & 90.4 & 45.2 & 41.6 & 344 & 31.34 & 213 & 135.8 & 248 & 42 & 14.8 & 9.7 & 2.16 & 0.0 & 148 & 49831 & 100320 \\
\hline $3 \mathrm{r}$ & 23.5 & 00 & 34 & 10.5 & 5.80 & 19 & 27.0 & 0.0 & 00 & 0.0 & 0.0 & 00 & 00 & 0.0 & 7206 & 1.4508 \\
\hline $\mathbf{n}$ & 34 & 0.0 & 0.4 & 2.5 & 0.45 & 00 & 1.2 & 0.2 & 0.7 & 01 & 0.0 & 00 & 024 & 00 & 939 & 0.1391 \\
\hline$\pi$ & 59 & 0.0 & 00 & 0.0 & 014 & 00 & 0.0 & 0.0 & 00 & 0.0 & 0,0 & 0.0 & 0.23 & 00 & 623 & 0.1254 \\
\hline$\pi$ & 17 & 00 & 20 & 0,0 & 0.0 & 0.0 & 80 & 0.9 & 00 & 0.0 & 0.0 & 0.91 & 20 & 0.41 & 3.03 & 0,0811 \\
\hline $\mathrm{In}$ & 10 & 0.0 & 00 & 0.0 & 0.0 & 0.0 & 00 & 0.0 & 00 & 0.0 & 0.0 & 0.0 & 00 & 00 & 0.95 & 0.0192 \\
\hline W & 29 & 09 & 16 & t.4 & 150 & 1.7 & 2.5 & 12 & 10 & 1.3 & 1.0 & 0.0 & 030 & 0.0 & 1731 & 0.3485 \\
\hline $\mathrm{Zx}_{\mathrm{x}}$ & 136.4 & 429 & 59.4 & 880 & 98.90 & 840 & 124.3 & 75.8 & 801 & 87.2 & 52.0 & 0.0 & 092 & 250 & 94211 & 189668 \\
\hline z & 00 & 0.0 & 00 & 0.0 & 0.0 & 0.0 & 0.0 & 0.0 & 0.0 & 0.0 & 0.0 & 8.04 & 6.20 & 121 & $1 \times 49$ & 03110 \\
\hline $\begin{array}{c}\text { grcuntote } \\
\text { wil }\end{array}$ & 59029 & 286.67 & 304.24 & 258.92 & 24932 & 416.79 & 260.71 & 405.66 & 563.85 & 363.92 & $107 \times 0$ & 288.62 & 1500 & $12 \%$ & $4967,1 ?$ & \\
\hline Grand $\mathrm{S}$ & 11.884 & -5.7312 & 6.1251 & 6.018 & 5.8246 & 8.391 & $14+107$ & 1.1682 & 17391 & 7719 & 2.1683 & 5.8106 & 0,3627 & 0.3809 & & \\
\hline
\end{tabular}

\section{References}

1. L. E. Feinendegen and K. Kasperek, Trace Element Analytical Chemistry in Medicine and Biology, P. Bratter and P. Schramel, eds., Proc. 1st international Workshop Neuherberg, FRG, (1980) 1-19.

2. E. J. Underwood, Trace Elements in Human and Animal Nutrition, (4th ed., Academi) (1977).

3. G. Ingrao, P. Belloni, S. Di Pietro, AND G. P. Santaroni . Levels of Some Trace Elements in Selected Autopsy Organs and in Hair and Blood Samples from Adult Subjects of the Italian Population, The Humana Press Inc (1990) 699-708

4. N. Suttle Mineral Nutrition of Livestock, 4th Edition, 2010. Br. Lib, Lon., UK., 579 pp

5. N. B. Ivanenko, A. A. Ganeev, N. D. Solovyev, and L. N. Moskvin. Determination of Trace Elements in Biological Fluids. J. Anal. Chem, 66(9), (2011) 784-799

6. R.K. Murray, D.K. Granner, P.a. Mayes, V. W. Rodwell. Harper's illustrated Biochemistry. Lange Medical Books/McGraw-Hill (2003) 677pp.
7. C. H. Evans. Biochemistry of the Lanthanides, Springer Science+Business Media, NY, (1990) 444 pp

8. Maret W. The Metals in the Biological Periodic System of the Elements: Concepts and Conjectures, Int. J. Mol. Sci. 2016, 17, 66; doi:10.3390/ijms17010066

9. B.A. Ali, M.M.M. Ahmad, M. Bahie EL-Deani and H. M. Shalan., Egypt. Poult. Sci. 22 (1) (2002) 59- 71

10. D. Huss, G. Poynter , R Lansford. Japanese quail (Coturnix japonica) as a laboratory animal model. LAB ANIMAL, Vol37, No. 11, (NOVEMBER 2008) 513-519

11. Clum N.J. Nutrient Content of five species of domestic animals commonly fed to captive raptors, J. Raptor Res. 31 (3)(1997)267-272

12. J. Sales, M. Skřrivan and M. Englmaierová. Influence of animal age on body concentrations of minerals in Japanese quail (Coturnix japonica), J. Anim. Phys. Anim.lNutr. 98 (2014) 1054-1059 (C) 2014 Blackwell Verlag $\mathrm{GmbH}$

13. G. R. Donia. Determination of Some Heavy Elements Residues in Some Organs of

Arab J. Nucl. Sci. \& Applic. Vol. 53, No.3 (2020) 
Migratory Quail in Relation to Public Health, Vol. 4, No. 10, October (2015) 2048-2059

14. N. N. Khomichenko, A. V. Shaverina, A. R. Tsygankova, and A. I. Saprykin. Inorganic Materials, Vol. 52, No. 14, (2016) 1405-1412.
15. A.M.Abdelhady, S.A. Abd El Aal, M.M.ElOkr and A.I.Helal. Arab J. Nucl. Sci. App. 50 (2) (2017)

16. NRC,1994. National Research Council. Nutrient requirements of poultry. 9th ed. rev. Washington: National Academy Press;. 